
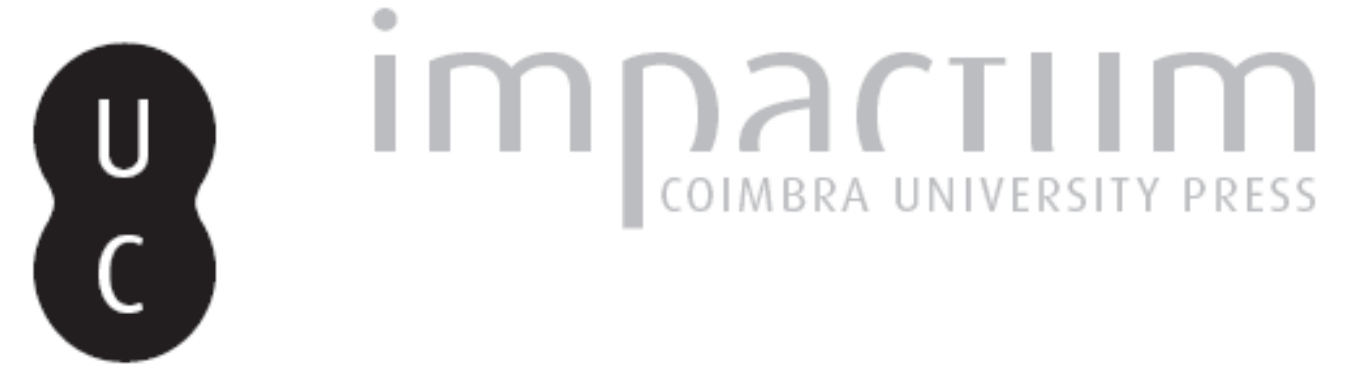

\title{
Facultés et parties de l'âme chez Platon
}

Autor(es): Delcomminette, Sylvain

Publicado por: Imprensa da Universidade de Coimbra

URL persistente: URI:http://hdl.handle.net/10316.2/42210

DOI: $\quad$ DOI:https://doi.org/10.14195/2183-4105_8_4

Accessed : $\quad$ 26-Apr-2023 14:08:54

A navegação consulta e descarregamento dos títulos inseridos nas Bibliotecas Digitais UC Digitalis, UC Pombalina e UC Impactum, pressupõem a aceitação plena e sem reservas dos Termos e Condições de Uso destas Bibliotecas Digitais, disponíveis em https://digitalis.uc.pt/pt-pt/termos.

Conforme exposto nos referidos Termos e Condições de Uso, o descarregamento de títulos de acesso restrito requer uma licença válida de autorização devendo o utilizador aceder ao(s) documento(s) a partir de um endereço de IP da instituição detentora da supramencionada licença.

Ao utilizador é apenas permitido o descarregamento para uso pessoal, pelo que o emprego do(s) título(s) descarregado(s) para outro fim, designadamente comercial, carece de autorização do respetivo autor ou editor da obra.

Na medida em que todas as obras da UC Digitalis se encontram protegidas pelo Código do Direito de Autor e Direitos Conexos e demais legislação aplicável, toda a cópia, parcial ou total, deste documento, nos casos em que é legalmente admitida, deverá conter ou fazer-se acompanhar por este aviso.

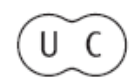




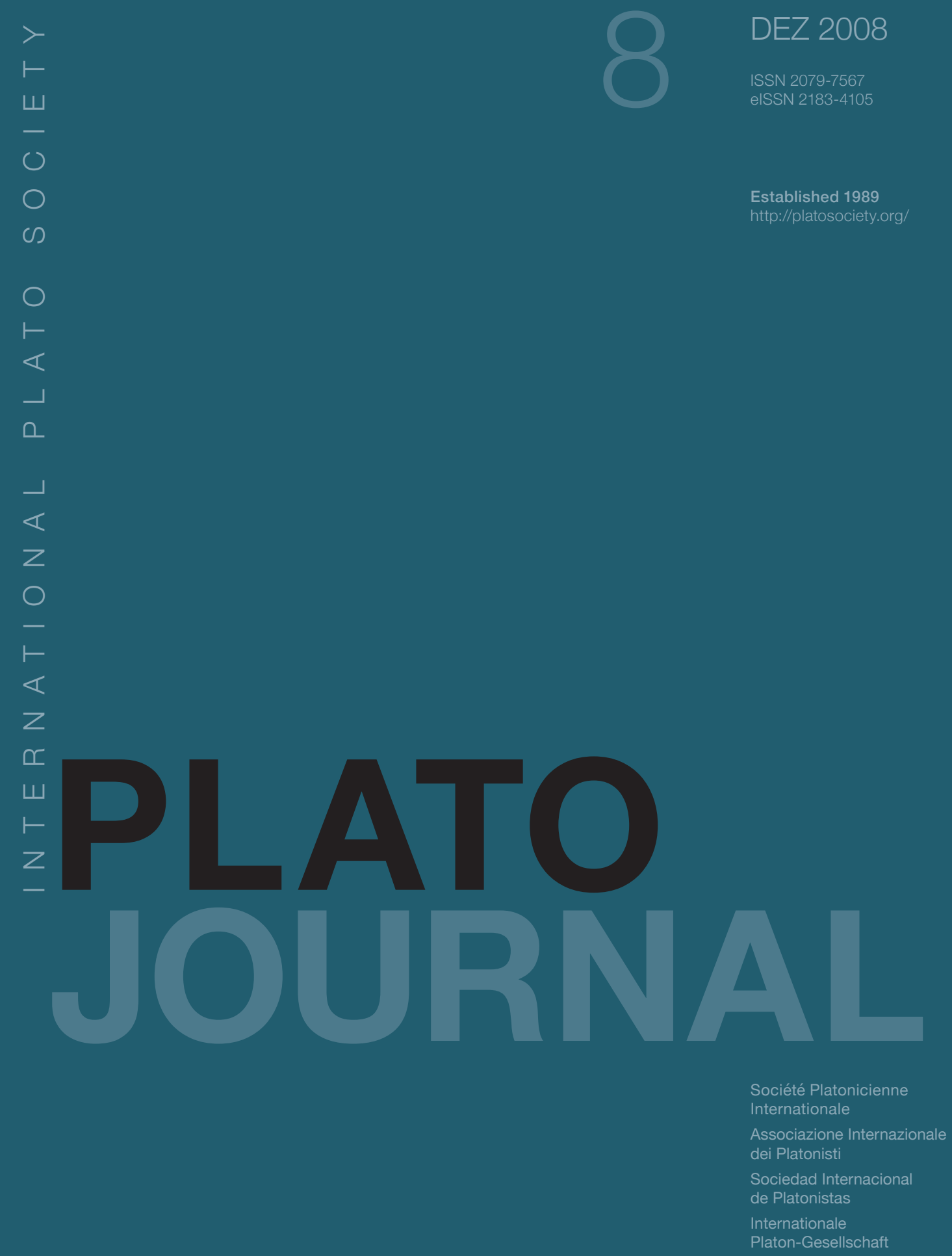




\section{FACULTÉS ET PARTIES DE L’ÂME CHEZ PLATON*}

Que désignent les expressions «partie de l'âme » et «faculté » chez Platon ? Traditionnellement, depuis Aristote au moins, ces deux expressions ont été considérées comme synonymes, et la plupart des interprètes modernes continuent, le plus souvent sans s'interroger véritablement sur cette question ${ }^{1}$, à assumer qu'elles renvoient à une seule et même notion. Je voudrais montrer ici que cette identification n'est pas justifiée et repose sur une confusion entre deux problématiques radicalement différentes chez Platon. Pour ce faire, je me baserai essentiellement sur le dialogue où ces deux notions sont instituées pour la première fois dans leur sens philosophique, à savoir la Républiqué2. Cependant, avant d'examiner les passages décisifs à cet égard, il convient de rappeler brièvement la manière dont Platon définit l'âme elle-même dans sa nature propre.

\footnotetext{
* Les références à la République se basent sur la nouvelle édition de S. R. SLINGS, Platonis Rempublicam, Oxford, Oxford Classical Texts, 2003. Pour les autres Dialogues, l'édition utilisée est la suivante : Platonis Opera, Oxford, Oxford Classical Texts, Vol. I, 1995 (éd. E. A. DuKe, W. F. Hicken, W. S. M. Nicoll, D. B. Robinson et J. C. G. STRACHAN) et Vol. II-V, 1901-1907 (éd. J. BURNET). Les extraits cités ont été retraduits ; cependant, en ce qui concerne la République, je me suis souvent inspiré de l'excellente traduction de P. PACHET (Paris, Gallimard, Collection « Folio », 1993).

${ }^{1}$ Par exemple, on ne trouve nulle part de discussion sur ce point dans l'ouvrage classique de T. M. ROBINSON, Plato's Psychology, Toronto, 1995. J'ai étudié les raisons qui ont pu pousser Aristote à identifier parties et facultés dans une communication au colloque sur la psychologie d'Aristote qui s'est tenu à Padoue en mars 2008.

2 J'invoquerai très peu ici le Timée, qui mériterait un traitement à part, car c'est ce dialogue qui doit selon moi être interprété à partir de la République et non l'inverse : en effet, c'est dans la République que les notions de parties et de facultés sont définies et étudiées pour elles-mêmes. J'espère y revenir en une autre occasion.
}

PLATO, The electronic Journal of the International Plato Society, n 8, 2008. http://gramata.univ-paris1.fr/Plato

(c) All rights of reproduction of any form reserved. 


\section{Définition de l'âme}

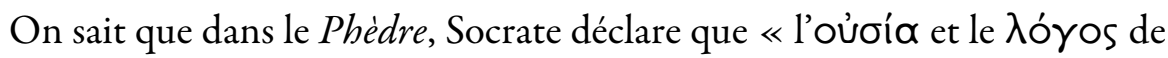
l'âme » (245 e3-4) est « ce qui se meut par soi-même » (Tò aúTò Kıvoũv, 245

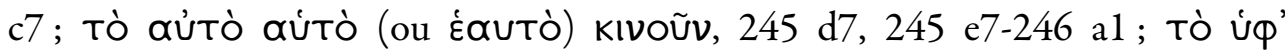

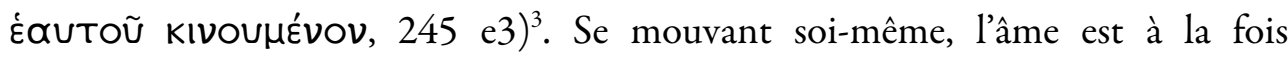
mouvante et mue. Mais cela ne nous oblige-t-il pas à distinguer en elle deux parties, l'une qui meut et l'autre qui est mue ${ }^{4}$ ? C'est ce qu'affirmera Aristote dans le cinquième chapitre du livre VIII de la Physique, en en tirant la conclusion que le premier moteur (ou mouvant) doit nécessairement être quant à lui immobile. Cependant, une telle conséquence ne s'avère nécessaire que si l'on considère «ce qui se meut par soi-même » comme une chose qui se meut. Or tel n'est précisément pas le cas de Platon, qui dans le dixième livre des Lois reformule le

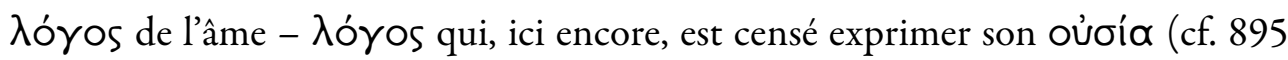
d4) - en la désignant à présent comme le mouvement capable de se mouvoir lui-

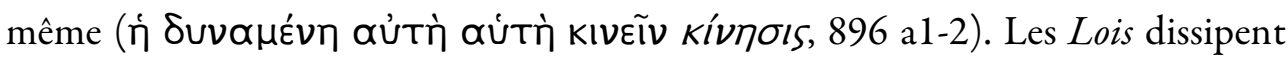
ainsi l'ambiguïté que laissait subsister le Phèdre ${ }^{5}$, en précisant que l'âme n'est pas une chose qui se meut, mais un pur mouvement, qui n'affecte aucun substrat autre

\footnotetext{
${ }^{3}$ L'expression tò aútokívntov, imprimée par L. RoBIN dans son édition du Phèdre (Platon : Phèdre, Paris, Les Belles Lettres, Collection des Universités de France, 1933) à la place de tò ó:ıikívๆTov retenu par Burnet en 245 c5, ne figure pas dans les manuscrits, mais dans un papyrus du début du $\mathrm{III}^{\mathrm{e}}$ s. après J.-C. Les deux expressions sont des hapax chez Platon. La décision entre ces deux leçons est importante pour la reconstruction de la preuve de l'immortalité de l'âme qui suit, mais nous pouvons l'ignorer ici, dans la mesure où la notion d'automotricité est quant à elle clairement véhiculée par les autres expressions citées ci-dessus.

${ }^{4}$ Comme l'écrit R. BETT, «Immortality and the nature of the soul in the Phaedrus », repris dans G. Fine (éd.), Plato. 2 : Ethics, Politics, Religion, and the Soul, Oxford, 1999, pp. $428-9$ et n. 8.

${ }^{5}$ Notons toutefois que dans le Phèdre, l'âme est comparée, non pas à un attelage ailé, comme on le

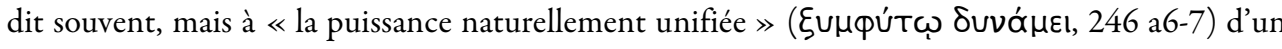
attelage ailé et d'un cocher, ce qui suggère déjà qu'elle n'est rien d'autre que « puissance » - cette puissance étant précisément le fait de se mouvoir soi-même, comme nous l'apprenons plus loin (246c4).
}

PLATO, The electronic Journal of the International Plato Society, n 8, 2008.

http://gramata.univ-paris1.fr/Plato

(c) All rights of reproduction of any form reserved. 
que lui-même $e^{6}$. De ce mouvement, il ne faut chercher la cause nulle part ailleurs qu'en lui-même : en l'âme, cause et effet ne font qu'un, ce qui meut et ce qui est mû s'identifiant purement et simplement. C'est en tant qu'elle est elle-même un mouvement que l'âme est principe de tous les autres mouvements ('́م)

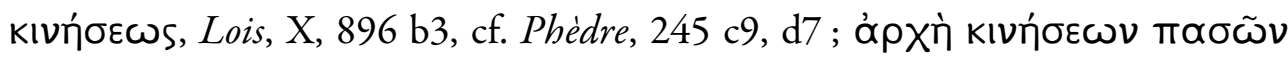

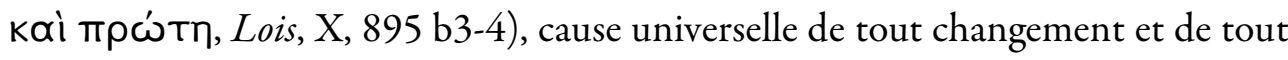

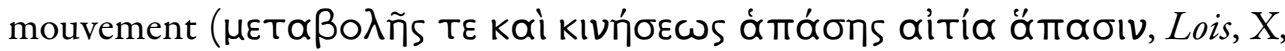
896 b1).

Comment concevoir un tel mouvement ? L'Athénien des Lois nous le dit explicitement : les mouvements de l'âme ne sont pas des mouvements physiques mouvement circulaire, translation, division, composition, croissance, décroissance, génération et corruption (cf. X, 893 b1-894 a8) -, même s'ils en sont les principes; ils ont pour noms « vouloir, examiner, prendre soin, délibérer, opiner correctement ou faussement - se réjouissant ou étant peinée, étant confiante ou craintive, haïssant ou chérissant, et tous les mouvements apparentés à ceux-là » $(\mathrm{X}, 897 \mathrm{a} 1-4)^{7}$. On pense à la définition cartésienne de la res cogitans

\footnotetext{
${ }^{6} \mathrm{R}$. DEmOS insiste avec raison sur ce point fondamental: « The point to stress is that there are not two things : the soul, on the one hand, and the soul's activity, on the other. The logical distinction between subject and predicate has no place here ; to be a soul is to be an activity » (« Plato's doctrine of the psyche as a self-moving motion », Journal of the History of Philosophy, 6, 1968, p. 137). Cette conception de l'âme ne me semble nullement incompatible avec celle du Phédon, qui aboutit finalement à identifier l'âme avec la vie elle-même, comme j’ai tenté de le montrer dans «Vie "biologique" et vie "morale" chez Platon », à paraitre dans les Cabiers $d u$ Centre d'études sur la pensée antique « kairos kai logos».

${ }^{7}$ Ce texte devrait suffire à montrer combien les arguments d'Aristote contre la mobilité de l'âme en De Anima, I, 3 ne sont nullement décisifs contre la position de Platon, le mouvement attribué à l'âme par ce dernier n'appartenant à aucune des espèces de mouvements reconnues par Aristote. Dire, avec E. N. OsTENFELD (Forms, Matter and Mind. Three Strands in Plato's Metaphysics, La Haye, 1982, pp. 263-4), que ce passage des Lois n'implique pas encore que le mouvement psychique soit conçu comme non physique, voire qu'il suggère le contraire, sous prétexte que ce mouvement est désigné comme la cause de tous les mouvements physiques, me paraît inconciliable avec le texte, qui décrit précisément les mouvements de l'âme pour les opposer aux mouvements

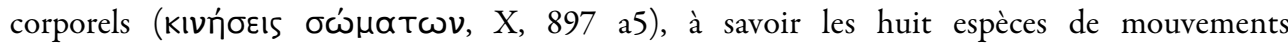
précédemment cités. En effet, quels mouvements physiques pourraient subsister en dehors du
}

PLATO, The electronic Journal of the International Plato Society, n 8, 2008.

http://gramata.univ-paris1.fr/Plato

(c) All rights of reproduction of any form reserved. 
comme « une chose qui doute, qui conçoit, qui affirme, qui nie, qui veut, qui ne veut pas, qui imagine aussi, et qui sent $\gg^{8}$, sinon que le grec permet à Platon d'éviter d'utiliser le terme « chose ». Selon ce texte des Lois, les mouvements psychiques correspondent donc à ce que nous appellerions aujourd'hui des mouvements de conscience, mouvements en lesquels la distinction entre ce qui meut et ce qui est mû n'a effectivement plus lieu d'être, la conscience étant mue dans la mesure même où elle meut, et inversement. Quant à l'âme elle-même, elle n'est pas une entité distincte de ces mouvements, qui se cacherait en quelque sorte « derrière » chacun d'eux, mais elle s'identifie avec ces mouvements eux-mêmes, ou plus exactement avec la matrice de tous ces mouvements, à savoir le mouvement qui se meut soi-même en tant que tel. C'est à la lumière de cette conception de l'âme comme mouvement qui se meut soi-même qu'il faut tâcher de comprendre les notions de « parties » et de « facultés » de l'âme.

\section{Les parties de l'âme}

a) Qu'est-ce qu'une partie de l'âme?

\footnotetext{
mouvement circulaire, de la translation, de la division, de la composition, de la croissance, de la décroissance, de la génération, de la corruption et des altérations qui en résultent ? Certes, dans le Timée, le mouvement de l'intelligence est représenté par un mouvement circulaire; mais précisément, l'Athénien des Lois dit explicitement qu'il s'agit seulement là de l'image du mouvement de l'intelligence (cf. Eikóva, X, 897 e1 ; sur le sens de cette image, voir E. N. LEE, « Reason and rotation: Circular movement as the model of mind (nous) in later Plato », dans W. H. WerkMEISTER (éd.), Facets of Plato's Thought, Assen/Amsterdam, 1976, pp. 70-102). Or

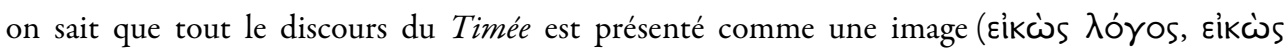
$\mu \tilde{\theta} \theta 0$ ).

${ }^{8}$ Descartes, Méditations métaphysiques, Méditation Seconde, dans CEuvres philosophiques, édition de F. ALQUIÉ, T. II, Paris, 1983, pp. 420-1.
}

PLATO, The electronic Journal of the International Plato Society, n 8, 2008. http://gramata.univ-paris1.fr/Plato

(c) All rights of reproduction of any form reserved. 
Si l'âme est un pur mouvement, en quel sens peut-on dire qu'elle se divise en parties? Afin de répondre à cette question, il convient d'examiner attentivement l'argumentation du livre IV de la République où Socrate établit la tripartition de l'âme ( 434 d1 sq.).

Remarquons tout d'abord que le terme $\mu$ épos apparaît à la fois très rarement et très tardivement dans ce passage: on n'en trouve que trois occurrences en ce sens dans le livre IV, en 442 b10, 442 c 4 et 444 b3. Il réapparaît en revanche plusieurs fois dans le livre IX (cf. 577 d4, 581 a6, 586 e5). Par luimême, il n'indique encore rien sur la nature de ces parties, étant donné l'usage extrêmement large qu'en fait Platon : par exemple, au livre VI (495 a5), on trouve

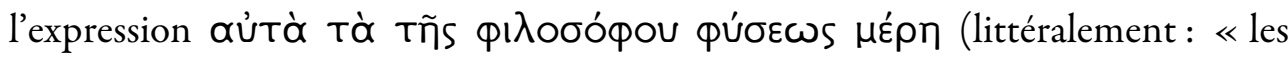
parties elles-mêmes qui constituent le naturel philosophe $\gg)$, pour désigner toutes les vertus et tous les traits de caractère qui définissent un naturel philosophe (voir aussi 503 b8).

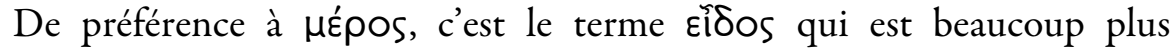
fréquemment utilisé dans le livre IV pour désigner les parties de l'âme (cf. $435 \mathrm{cl}$, c5, e1, 439 e1, e6, e7 ; voir également VI, 504 a 4 et X, 595 b1). Ce terme est intéressant, car il correspond au terme $\gamma \varepsilon ́ v o s$, utilisé quant à lui pour désigner les trois catégories d'hommes que l'on peut distinguer dans la cité idéale. Cette correspondance, exigée par l'analogie entre la cité et l'âme, est clairement indiquée dès la position du problème en 435 b4-c3, et répétée notamment en 440 e6-441


parties de l'âme elles-mêmes (cf. 441 c6, 443 d3), tandis qu'Eỉరos remplace parfois Yévos dans le cas de la cité (par exemple en 435 e 1 et en IX, 580 d2).

Or dans la cité, le terme yévos désigne un ensemble d'hommes - ou plus

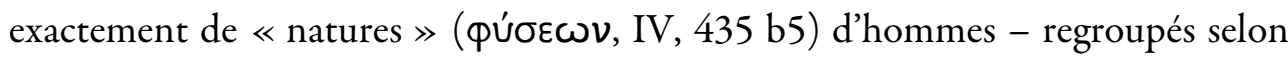

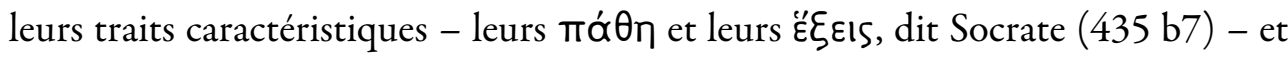
formant une «classe » qui, parce qu'elle ne comprend qu'une partie des citoyens, peut également être nommée une « partie » de la cité (cf. 428 e7, 429 b2, b8, 431 e10). Les parties de la cité sont donc des genres regroupant chacun une multiplicité d'individus, multiplicité d'ailleurs variable selon les genres considérés.

PLATO, The electronic Journal of the International Plato Society, n 8, 2008. http://gramata.univ-paris1.fr/Plato

(c) All rights of reproduction of any form reserved. 
En vertu de l'analogie entre la cité et l'âme, les عî́ర̄ de l'âme devraient dès lors eux aussi être considérés non comme des entités individuelles, mais comme des espèces regroupant chacune une multiplicité. Mais une multiplicité constituée de quels éléments? Pas plus que la cité ne consiste en une multiplicité de cités, l'âme n'est faite d'une multiplicité d'âmes'. Les trois espèces ne sont donc pas, de prime abord du moins, trois espèces d'âmes, même si elles sont trois espèces de l'âme. Mais l'âme n'est pas une entité figée et monolithique : elle est un mouvement qui se meut soi-même, et qui se manifeste d'emblée sous la forme d'une multiplicité de mouvements différents. C'est cette multiplicité de mouvements psychiques qu'il s'agit de rassembler au sein de trois espèces distinctes.

Cependant, les parties de l'âme ne sont pas identifiées à la simple somme des mouvements appartenant aux espèces en question, mais bien plutôt aux principes de ces mouvements. Cela apparaît clairement lorsque Socrate pose le

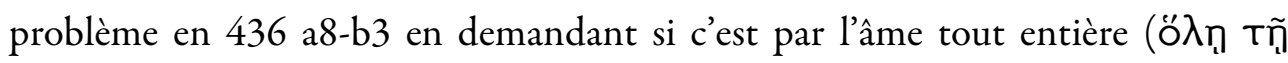
$\cup \times \tilde{n})$ que nous accomplissons chacune de nos actions, «à chaque fois qu'un

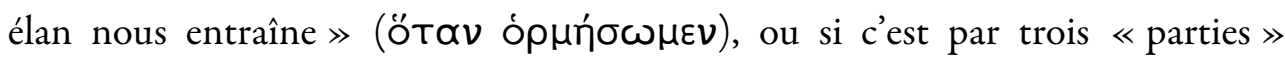
différentes que nous apprenons ( $\mu \alpha \nu \theta \alpha \dot{\nu} O \mu \varepsilon v)$, que nous avons de l'ardeur $(\theta \cup \mu \circ u ́(\mu \varepsilon \theta \alpha)$ et que nous désirons les plaisirs liés à la nourriture et à la

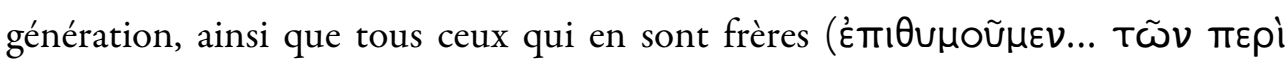

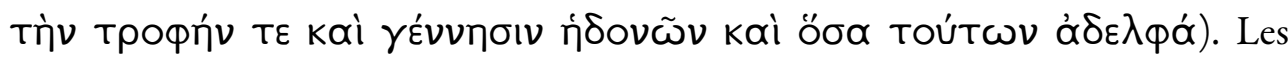
parties de l'âme sont donc destinées à expliquer les différents élans (óp $\mu \alpha i ́)$ qui

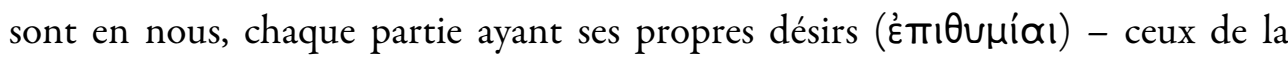

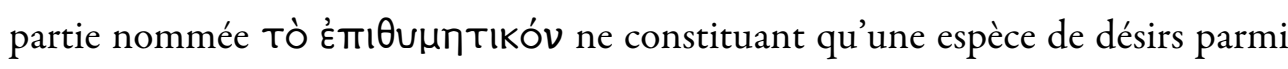
d'autres - et pouvant dès lors être considérée comme une ảpXń, c'est-à-dire comme un principe d'action, et donc de mouvement (cf. IX, 580 d6-7). Il n'y a toutefois aucune raison de comprendre de tels principes comme des entités qui se cacheraient « derrière » les mouvements qu'ils suscitent, sortes de moteurs qui demeureraient quant à eux immobiles, car si l'âme est un mouvement, rien en elle

\footnotetext{
${ }^{9}$ Nous verrons toutefois que ces affirmations doivent être nuancées, tant dans le cas de la cité que dans le cas de l'âme.
}

PLATO, The electronic Journal of the International Plato Society, n 8, 2008. http://gramata.univ-paris1.fr/Plato

(c) All rights of reproduction of any form reserved. 
ne peut être immobile; ils sont bien plutôt principes de mouvement au sens de l'âme elle-même, c'est-à-dire en tant que mouvements qui se meuvent eux-mêmes. En d'autres termes, tout comme l'âme dans sa totalité, ses parties sont des mouvements automoteurs qui peuvent être considérés comme les matrices de mouvements psychiques; mais simplement, alors que l'âme elle-même joue ce rôle à l'égard de tous les mouvements psychiques, chacune de ses parties est liée à une partie seulement de ces mouvements, regroupés au sein d'une espèce unique en raison de l'identité de leur structure, celle-ci correspondant précisément au principe à l'œuvre en chacun d'eux. Le principe des différentes espèces de mouvements psychiques n'est donc pas une «instance» distincte et indépendante de ces mouvements eux-mêmes, mais seulement la structure qui s'exprime en chacun des mouvements qui appartiennent à cette espèce, et qui ne peut s'exprimer qu'en eux ${ }^{10}$.

Cette interprétation me paraît s'imposer dès lors que l'on prend au sérieux la définition platonicienne de l'âme comme «mouvement qui se meut soimême » et que l'on résiste à la tentation de la réifier pour en faire une «chose qui se meut soi-même ». Les seules parties que puisse avoir un mouvement sont des mouvements, et si ces mouvements sont des mouvements psychiques, ils doivent se mouvoir eux-mêmes. De plus, cette interprétation permet de maintenir la cohérence de l'analogie entre l'âme et la cité, car les « genres d'hommes » qui composent cette dernière ne reposent pas sur des «principes » extérieurs aux hommes qui les constituent, mais seulement sur des traits qui les caractérisent tous semblablement. Notons toutefois qu'elle semble mener à un paradoxe : si les

\footnotetext{
${ }^{10}$ En ce sens, je m'écarte de G. SANTAS qui, ayant repéré cette distinction entre les espèces de mouvements psychiques et leurs principes, en conclut que la division de l'âme en parties repose sur l'assomption non dite selon laquelle les activités psychiques d'une espèce donnée impliquent des facultés qui en sont principes (Goodness and Justice. Plato, Aristotle, and the Moderns, Oxford, 2001, pp. 118-19). Il me semble au contraire que cette distinction n'implique pas l'existence d'une quelconque «instance » autre que ces mouvements eux-mêmes, sous peine de contredire la définition de l'âme comme pur mouvement. Par ailleurs, nous verrons plus loin que les parties de l'âme ne peuvent en aucun cas être confondues avec des «facultés», surtout si l'on entend ce terme au sens que lui donne la fin du livre V de la République, comme le fait G. Santas (cf. p. 122).
}

PLATO, The electronic Journal of the International Plato Society, n 8, 2008.

http://gramata.univ-paris1.fr/Plato

(c) All rights of reproduction of any form reserved. 
parties de l'âme sont elles-mêmes des mouvements qui se meuvent eux-mêmes, ne pourrait-on pas les considérer comme des âmes à part entière, de sorte que les espèces de l'âme seraient en même temps des espèces d'âmes, contrairement à ce que nous avons suggéré plus haut? Aussi étrange qu'elle puisse paraitre, cette conséquence semble bel et bien reconnue et acceptée par Platon, qui, dans le Timée, n'hésite pas à désigner les parties de l'âme, non pas seulement comme des

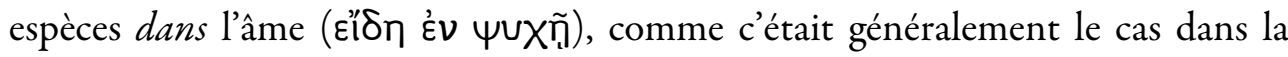
République, mais comme des « espèces d'âme » (voir notamment $69 \mathrm{c} 7$ : દỉరos...

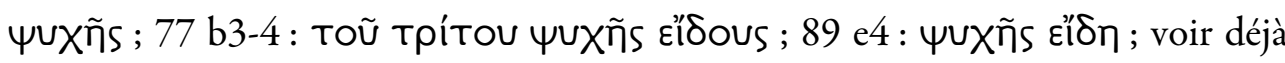

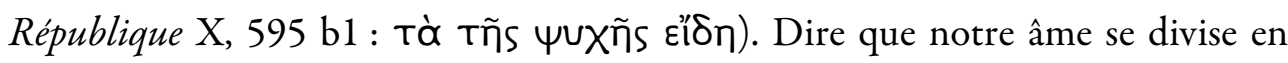
trois parties signifierait-il donc que nous avons trois âmes? Nous verrons plus loin en quel sens il convient de nuancer cette proposition.

\section{b) Distinction des parties de l'âme}

Reste à comprendre ce qu'il faut entendre par ces «structures » qui soutiennent chacune des trois espèces de mouvements psychiques. À cette fin, examinons de plus près le texte du livre IV de la République où les parties de l'âme sont distinguées pour la première fois. Le principe général sur lequel repose cette distinction est le suivant : «Il est évident que la même chose ne consentira pas à faire ou à subir en même temps des contraires, en tout cas pas selon le même rapport et relativement à la même chose $(\Delta \tilde{\eta} \lambda$ ov ötı TaủTòv TávavTía

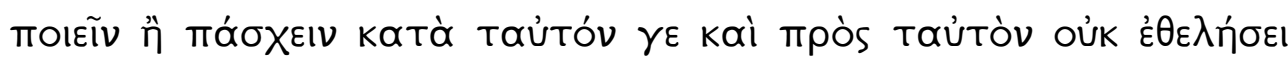
$\left.\alpha \mu^{\prime} \alpha\right)$; de sorte que si nous trouvons en quelque manière que cela se produit en eux [à savoir en ce par quoi nous apprenons, ce par quoi nous avons de l'ardeur et ce par quoi nous désirons les plaisirs liés à la nourriture et à la génération], nous saurons qu'ils ne sont pas un même principe, mais plusieurs » (436 b9-c2). Comme on l'a souvent remarqué, il s'agit ici d'une version du principe de contradiction, bien que les différences entre sa formulation et celles d'Aristote ait

PLATO, The electronic Journal of the International Plato Society, n 8, 2008. http://gramata.univ-paris1.fr/Plato

(c) All rights of reproduction of any form reserved. 
conduit certains interprètes à refuser cette identification ${ }^{11}$. Sans entrer ici dans les détails, insistons ici sur la présence de la restriction mpòs taútóv (« relativement à la même chose »), qui ne se trouve généralement pas dans les formulations d'Aristote ${ }^{12}$. Certes, on peut expliquer cette omission aristotélicienne en considérant qu'une telle précision est d'une certaine manière déjà comprise dans l'expression katà taưTóv ? («sous le même rapport »). Cependant, le fait que Socrate cite ces deux restrictions côte à côte suggère qu'il ne les tient pas quant à lui pour synonymes. Cela semble donner raison à Adam qui écrit que Kaтò TaủTóv signifie ici « dans la même partie » ${ }^{13}$. Quoi qu'il en soit, l'insistance de Socrate sur le fait que c'est « relativement à la même chose » que la même chose ne peut en même temps faire ou subir des contraires est tout à fait essentielle pour notre propos, car elle implique que les parties de l'âme vont être distinguées en fonction de leur attitude relativement à un seul et même objet. Cela suppose donc que les différentes parties de l'âme puissent entrer en contact avec le même objet, ce qui suffit déjà à les distinguer des facultés, dont nous verrons qu'elles se différencient par le fait de porter sur des objets différents. Pour être amenés à distinguer différentes parties en l'âme, nous devons repérer en elle des tendances contraires qui coexistent en même temps, sous le même rapport et relativement au même objet.

Afin de déterminer ce qu'il en est de cet objet, Socrate introduit un second principe, tout aussi fondamental que le premier, mais dont la portée a souvent été mal comprise. On peut le nommer le principe de la détermination réciproque des corrélatifs. Socrate l'énonce comme suit : « parmi toutes les choses

\footnotetext{
${ }^{11}$ Voir par exemple R. ROBINSON, « Plato's separation of reason from desire », Phronesis, 16, 1971, pp. 38-40 ; J. MOLINE, « Plato on the complexity of the psyche », Archiv für Geschichte der Philosophie, 60, 1978, p. 3, n. 8 ; T. IRWIN, Plato's Ethics, Oxford, 1995, p. 381, n. 4. G. SANTAS, op. cit., p. 160, n. 12, est plus nuancé. Pour une défense récente de l'identité entre ce principe et celui de contradiction, cf. S. MACERI, « On the law of contradiction in Republic, $436 \mathrm{~b}-437 \mathrm{a}$ », Platon, 46, 1994, pp. 135-8.

${ }^{12}$ Elle apparaît toutefois en Réfutations sophistiques, 5, 167 a26.

${ }^{13}$ J. ADAM, The Republic of Plato, Edited with Critical Notes, Commentary and Appendices, Cambridge, 1902, Vol. I, p. 247, n. ad loc.
}

PLATO, The electronic Journal of the International Plato Society, n 8, 2008. http://gramata.univ-paris1.fr/Plato

(c) All rights of reproduction of any form reserved. 
qui sont telles qu'elles sont "de" quelque chose, celles qui sont déterminées sont "de" quelque chose de déterminé, me semble-t-il, tandis que chacune en elle-

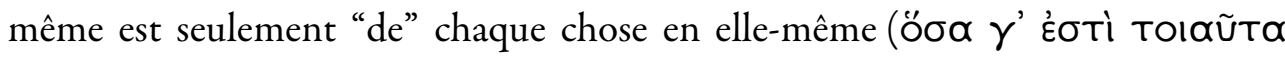

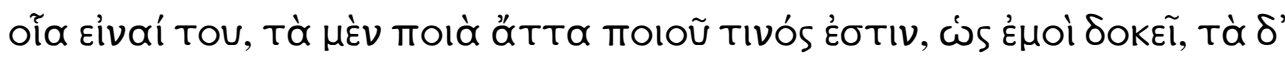

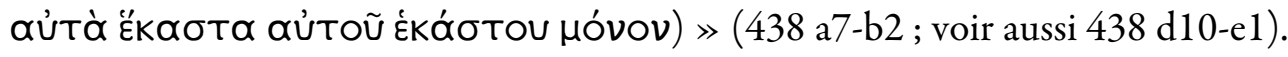

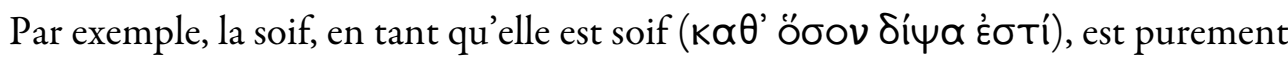
et simplement désir de boisson, et non d'une boisson déterminée de quelque façon que ce soit (437 c7-e8). Cela vaut même pour des déterminations aussi fondamentales que « bon » et « mauvais », car s'il est vrai que tous désirent ce

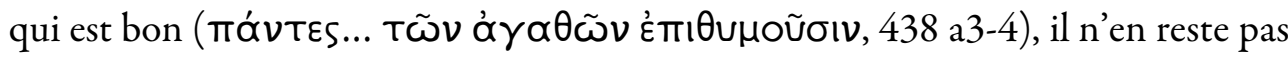
moins que la soif en tant que telle n'est pas plus désir d'une bonne que d'une mauvaise boisson (439 a4-b2).

On considère parfois ce passage comme le signe d'un renoncement dans le chef de Platon à la thèse socratique selon laquelle tout désir est désir du bien ${ }^{14}$. Cette thèse est pourtant réaffirmée plus loin dans le dialogue (cf. VI, 505 e1-2), et lorsque Socrate la cite dans le passage résumé ci-dessus, il ne paraît pas du tout la rejeter. On peut au contraire penser que l'énonciation de ce principe ne s'impose précisément que parce que cette thèse fondamentale demeure présupposée. En effet, si tout homme désire le bien à tout moment, comment expliquer la possibilité de désirs contraires? Ne parlons pas encore pour l'instant du type de conflits dont il sera question par la suite, mais tout simplement du fait que, par exemple, nous désirons tantôt boire et tantôt ne pas boire, ou que des personnes différentes peuvent désirer des choses contraires dans les mêmes circonstances. Même le Socrate des premiers Dialogues reconnaîtrait que de tels désirs sont contraires les uns aux autres, ce qui ne l'empêcherait pas pour autant de les considérer les uns et les autres comme différentes expressions du désir du bien. Mais précisément, il faut ici appliquer le principe de détermination réciproque des corrélatifs : de même que le désir d'une boisson chaude peut être considéré en

\footnotetext{
${ }^{14}$ Voir notamment T. PENNER, « Thought and desire in Plato », dans G. Vlastos (éd.), Plato : A Collection of Critical Essays. 2 : Ethics, Politics, and Philosophy of Art and Religion, New York, 1971, pp. 106-8, et T. IRWIN, op. cit., pp. 206-11.
}

PLATO, The electronic Journal of the International Plato Society, n 8, 2008. http://gramata.univ-paris1.fr/Plato

(c) All rights of reproduction of any form reserved. 
tant que simple soif, et que de ce point de vue son objet n'est pas la boisson chaude, mais la boisson en tant que telle, de même n'importe quel désir peut être considéré en tant que pur et simple désir, et de ce point de vue son objet est le bien en tant que tel, dénué de toute autre détermination. En d'autres termes, en tant que pur et simple désir, tout désir est désir du bien, mais en tant que tel désir particulier, il est désir de tel objet particulier; et c'est seulement à ce second niveau que surgit la possibilité de conflits, car le bien ne nous apparaît pas toujours de la même manière, non seulement parce qu'il se manifeste différemment dans des circonstances différentes, mais aussi et surtout parce que nous sommes incapables de saisir suffisamment ce qu'il est et d'avoir une conviction ferme à son égard (cf. VI, 505 e2-4). La fonction de ce passage est dès lors d'amener notre attention à se porter exclusivement sur l'objet immédiat du désir considéré, afin que nous examinions s'il est possible de découvrir en nous également un élan en sens contraire relativement à ce même objet. Si tel est le cas, ces deux désirs contraires n'en continueront pas moins à viser tous deux le bien en leur fond ; mais celui-ci se manifestera à eux de deux manières différentes, ce qui donnera lieu à un conflit que nous ne pourrons expliquer qu'en attribuant ces deux désirs à deux « parties » de l'âme différentes.

S'appuyant sur l'exemple de la soif, Socrate commence par distinguer la

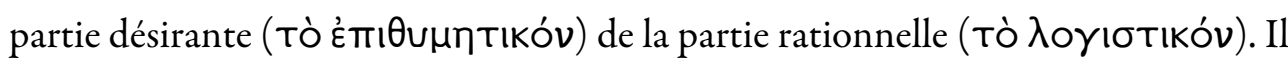
arrive souvent que, ayant soif, nous désirions boire, mais que pourtant, en vertu d'un raisonnement ('́k $\lambda \circ \gamma ı$ ○oũ), nous nous abstenions de boire. Dans ce cas, nous avons à la fois deux élans contraires sous le même rapport et relativement au même objet, ce qui, selon le principe énoncé plus haut, ne peut s'expliquer qu'à condition de reconnaître que ces élans proviennent de deux principes différents, l'un qui se contente de désirer et l'autre qui raisonne (IV, 439 b3-e2). Socrate

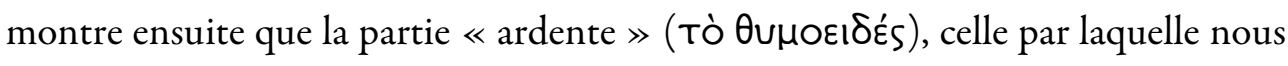

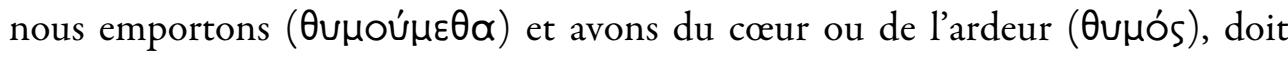
également être distinguée de la partie désirante, notamment en rappelant l'anecdote de Léontios qui, alors que son désir le poussait à regarder des cadavres

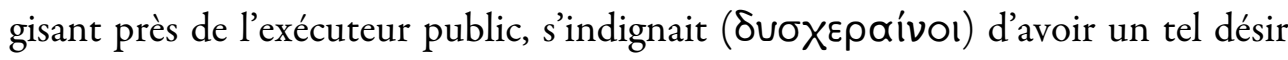

PLATO, The electronic Journal of the International Plato Society, n 8, 2008. http://gramata.univ-paris1.fr/Plato

(c) All rights of reproduction of any form reserved. 
et y résistait autant que possible, avant d'y céder finalement malgré sa profonde colère (ỏprí). Il remarque également que dans les cas de conflit interne de l'âme

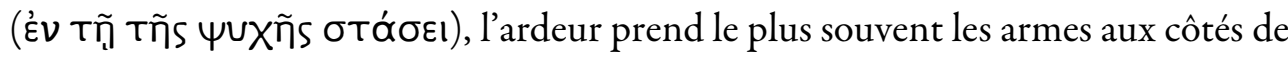
la partie rationnelle afin de combattre la partie désirante (439 e2-440 e5). Reste alors à montrer que l'ardeur est néanmoins distincte de la raison. Glaucon juge cela aisé, invoquant le fait que les jeunes enfants sont pleins d'ardeur dès leur

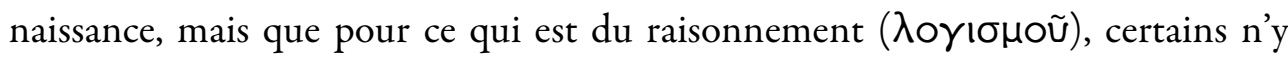

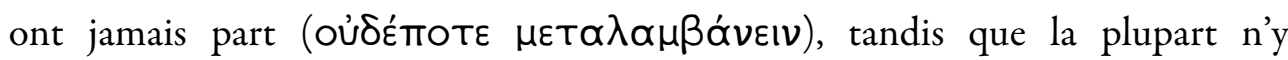
accèdent que tardivement ${ }^{15}$. Socrate renchérit en disant que tel est également le cas des bêtes ('̇̉ Toĩs Onpíoıs) et en citant un vers d'Homère dans lequel Ulysse réprimande son cœur par le biais de ce qui a raisonné sur le meilleur et sur le pire

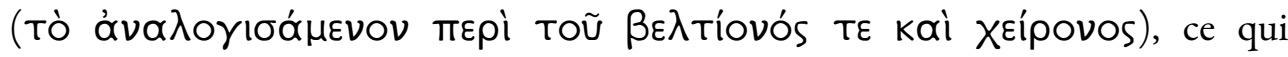
suppose qu'un conflit entre l'ardeur et la raison est également possible ${ }^{16}$ ( 440 e6441 c3). Nous pouvons donc conclure qu'il y a les mêmes « genres » dans l'âme de chacun que dans la cité, et qu'ils sont en nombre égal (441 c4-7) $)^{17}$.

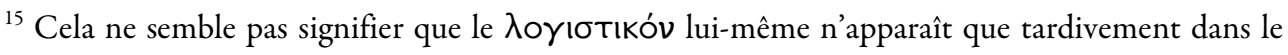
développement de la personne, mais plutôt que les jeunes enfants n'en font pas encore usage, c'est-

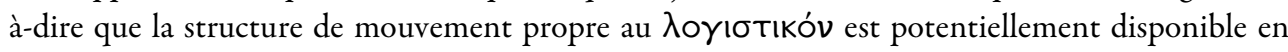
eux, mais n'a pas encore été déployée dans des mouvements effectifs. En effet, en VII, 518 c4-6, Socrate dit que la connaissance en tant que puissance est déjà présente en chacun de nous, ainsi que l'organe grâce auquel chacun apprend ; or nous verrons que cet « organe » n'est autre que le

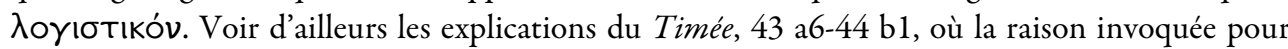

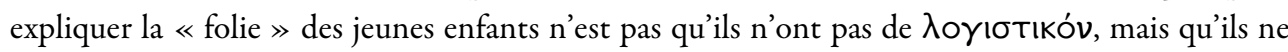
parviennent pas encore à en faire usage tant ils sont submergés par les mouvements suscités par le corps.

${ }^{16}$ Notons en effet que ce passage montre qu'il n'y a aucune raison de mettre en doute la possibilité

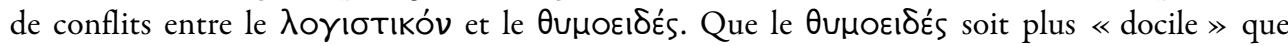

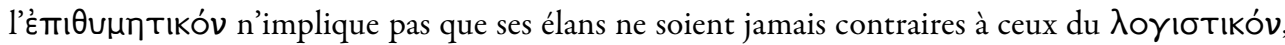

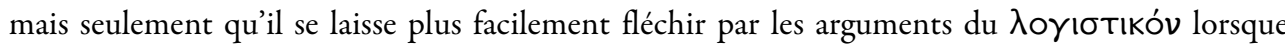
celui-ci tente de le remettre sur le droit chemin.

${ }^{17}$ On peut se demander ce qui garantit l'exhaustivité de cette division de l'âme, ainsi que ce qui guide Socrate dans la mise en évidence des conflits pertinents pour cette analyse. En réalité, il se règle sur la division précédente des genres d'hommes dans la cité, son but étant d'examiner si la même division se retrouve au sein de l'âme individuelle (cf. 435 b4-c3). Socrate est d'ailleurs le premier à reconnaître le caractère insuffisant de cette analyse purement empirique (cf. $435 \mathrm{c} 9$-d4),
}

PLATO, The electronic Journal of the International Plato Society, n 8, 2008.

http://gramata.univ-paris1.fr/Plato

(c) All rights of reproduction of any form reserved. 


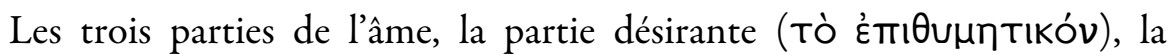

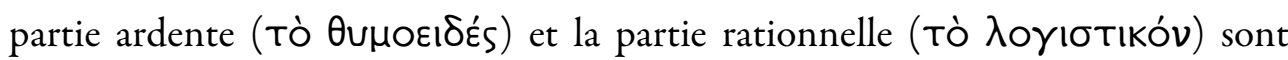
donc distinguées en fonction de l'attitude qu'elles adoptent à l'égard d'un seul et même objet, comme l'exige le principe sur lequel se fonde toute l'argumentation. Cependant, les indications données par Socrate au cours de cette analyse et des livres ultérieurs, en particulier les livres VIII et IX, permettent de préciser la nature de ces parties, en mettant au jour la structure des mouvements dont chacune est le principe. Chacune de ces structures comporte essentiellement deux aspects : un mode de fonctionnement particulier et un objet de désir qui lui est propre. Ces deux aspects ne sont d'ailleurs pas indépendants l'un de l'autre, l'objet du désir propre à chaque partie n'étant jamais que la figure sous laquelle le bien se manifeste à cette partie, figure qui est elle-même fonction du mode de fonctionnement de cette partie. En d'autres termes, l'objet du désir de chaque partie correspond à ce que cette partie peut atteindre du bien en vertu de son mode de fonctionnement propre. Notons toutefois d'emblée que cette liaison entre les parties de l'âme et les objets de leur désir n'est pas exclusive : elle ne signifie pas que chaque partie ne peut entrer en relation qu'avec son objet propre, sans quoi nous ne pourrions jamais observer des conflits entre différentes parties portant sur le même objet, mais seulement qu'elle juge tout le reste à l'aune de l'objet naturel de son désir, ce qui l'amène parfois à repousser ou à refuser l'objet du désir d'une autre partie lorsqu'il s'avère incompatible avec lui. Nous verrons que cette relation entre une partie de l'âme et l'objet de son désir se distingue de la relation entre une faculté et son objet, qui est quant à elle par définition exclusive.

et il n'exclut pas la possibilité de découvrir éventuellement d'autres parties « entre » celles qu'il a repérées (cf. $443 \mathrm{~d} 7$-e1).

PLATO, The electronic Journal of the International Plato Society, n 8, 2008. http://gramata.univ-paris1.fr/Plato

(c) All rights of reproduction of any form reserved. 


\section{c) Nature des trois parties de l'âme}

Tâchons à présent de caractériser brièvement les trois structures fondamentales de mouvements psychiques qui constituent les «parties» de l'âme ${ }^{18}$.

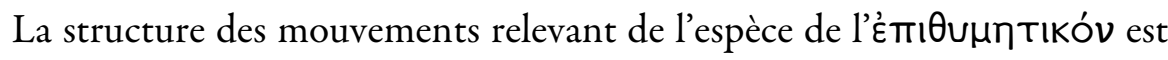
la plus simple. Son mode de fonctionnement est, non pas le désir en tant que tel, qui appartient aux mouvements des trois espèces, mais un désir « irrationnel »

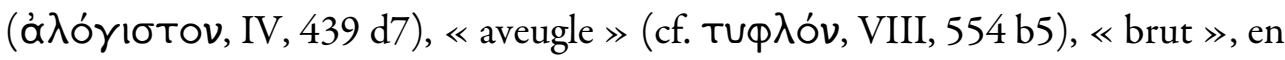
ce qu'il n'admet aucun intermédiaire entre lui-même et son objet, ni

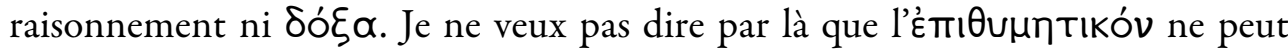
avoir de $\delta o ́ \xi \alpha$ - nous verrons plus loin que c'est bien plutôt le contraire qui est vrai -, mais seulement que celle-ci n'intervient pas dans la structure des désirs qui en dépendent - sinon peut-être à titre de résultat, en ce sens qu'il peut considérer que parce qu'il désire tel ou tel objet, celui-ci est nécessairement bon. C'est pourquoi cet objet est celui vers lequel nous porte le plus immédiatement notre désir, à savoir « les plaisirs liés à la nourriture et à la génération, ainsi que tous ceux qui en sont frères » (IV, 436 b1-2). Le livre IX ajoute à cette liste l'argent, « parce que c'est surtout au moyen de l'argent que de tels désirs se réalisent » (580 e5-581 a1). Cet ajout peut sembler faire problème : même si l'on comprend bien la liaison ici effectuée par Socrate entre désir de plaisir et désir d'argent, n'estil pas possible d'imaginer qu'ils entrent parfois eux-mêmes en conflit? Pour reprendre une objection déjà discutée par Proclus ${ }^{19}$, une personne qui serait à la fois gourmande et avare ne pourrait-elle pas expérimenter un conflit entre son désir de nourriture et son désir d'argent, en particulier lorsque lui sont présentés

\footnotetext{
${ }^{18}$ Les remarques proposées ci-dessous ne prétendent nullement épuiser la richesse de cette problématique. Pour une analyse plus complète, voir en particulier l'excellente étude de J. M. COOPER, « Plato's theory of human motivation » [1984], repris dans G. FINE (éd.), op. cit., pp. 186-206, dont je ne partage cependant pas toutes les conclusions (en particulier, je ne pense pas

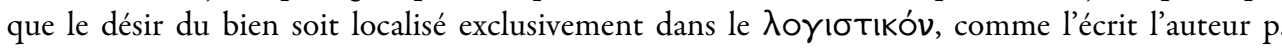
194).

${ }^{19}$ Proclus, Commentaire sur la République, VII ${ }^{e}$ Dissertation, 225.3-227.27 Kroll.
}

PLATO, The electronic Journal of the International Plato Society, n 8, 2008. 
des mets très coûteux? S'il en allait ainsi, selon le principe directeur de notre enquête, ne serions-nous pas obligés d'attribuer ces désirs à deux parties de l'âme différentes ? Cette conclusion ne semble pas s'imposer. En effet, le conflit a moins lieu entre deux désirs qu'entre un désir et le calcul des conséquences d'un autre désir. En vertu du principe de détermination réciproque des corrélatifs, l'objet propre du désir d'argent est purement et simplement l'argent, et celui du désir de nourriture la nourriture ; mais le rejet de la nourriture résulte d'un raisonnement sur les conséquences que son acceptation aurait sur la bourse de l'intéressé. Or ce

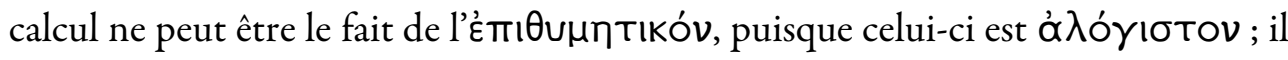

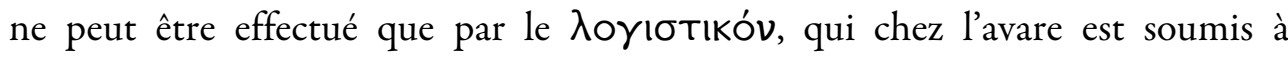

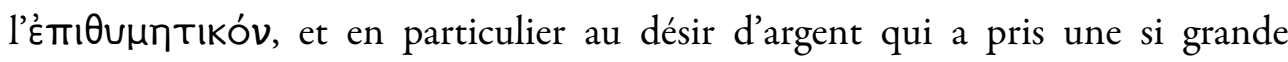
importance chez lui. Le conflit a donc bien lieu entre deux parties de l'âme différentes et non au sein d'un seule partie. Que les désirs de l'ĖmıӨuntıkóv nous tirent chacun dans des directions très diverses, Socrate est le premier à le reconnaître, puisqu'il compare cette partie de l'âme à une bête ayant de multiples têtes, certaines paisibles et d'autres sauvages (cf. IX, 588 c2-d1). Cependant, en eux-mêmes, ces désirs ne sont pas encore en conflit, car chacun tend seulement vers son objet immédiat - boisson, nourriture, argent, etc. - sans se préoccuper des autres. Les conflits peuvent seulement surgir lorsque sont envisagées les

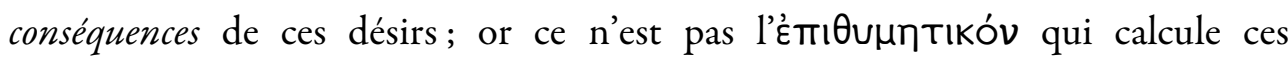

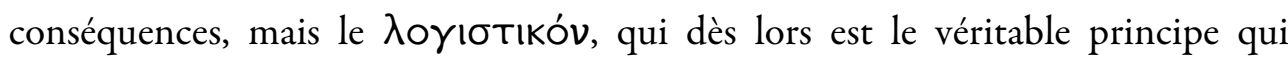
s'oppose à l'objet du désir considéré, même s'il le fait en fonction d'un autre désir auquel il se soumet.

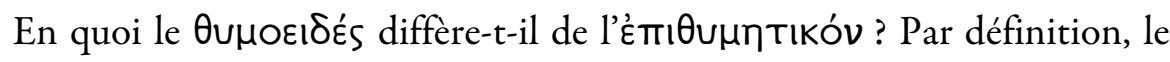

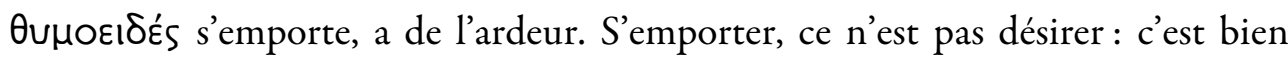
plutôt réagir face à une situation donnée, de manière irrationnelle certes (cf.

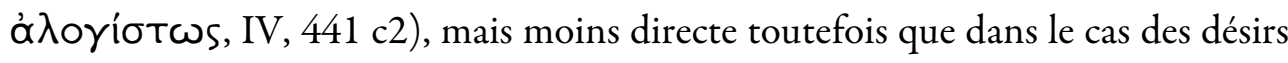
précédents. En effet, ce qui motive de tels comportements, c'est la recherche de la domination, de la victoire et des honneurs (cf. IX, 581 a9-b5). Or de telles valeurs sont essentiellement liées à la considération que les autres nous accordent, que

PLATO, The electronic Journal of the International Plato Society, n 8, 2008. http://gramata.univ-paris1.fr/Plato

(c) All rights of reproduction of any form reserved. 
celle-ci soit la raison pour laquelle nous les recherchons ou qu'elle en soit l'origine, et cette considération repose elle-même sur la $\delta o ́ \xi \alpha$ propre au groupe social en question relativement à ce qui est louable et à ce qui est blâmable. C'est parce qu'il a cette $\delta o ́ \xi \alpha$ en lui que le $\theta u \mu o \varepsilon ı \delta \varepsilon ́ s$ s'emporte, soit qu'elle lui semble bafouée dans la circonstance présente, soit qu'elle lui impose un certain type de comportement qui y soit conforme. Cela est clair dans le cas de Léontios, qui ne s'indigne de son désir de se repaître de la vue des cadavres que parce qu'il a en lui

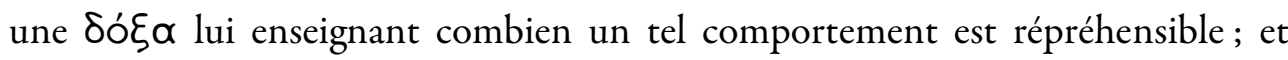
c'est plus clair encore lorsque Socrate dit que c'est seulement lorsque l'on considère (írñTaı) que l'on subit une injustice que l'on s'emporte et que l'on se

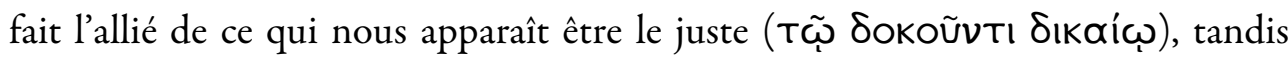

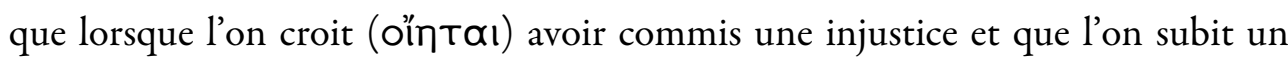
châtiment de la part de quelqu'un que l'on croit (ol̂nTđa) agir justement, on ne se

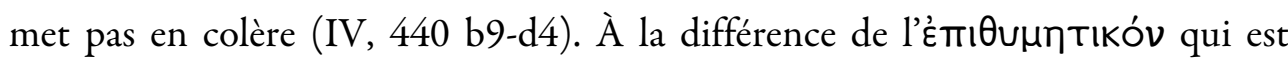
« aveugle », le $\theta u \mu o \varepsilon ı \delta \varepsilon ́ s$ réagit par rapport à une $\delta o ́ \xi \alpha$ qui lui dit ce qu’il doit faire, ce qui louable et ce qui est blâmable, et qui joue le rôle d'intermédiaire entre lui-même et l'objet de son désir. Bien entendu, il est tout à fait possible que cette

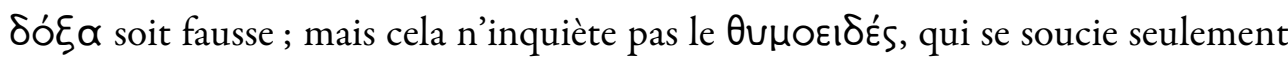
de la conformité de ses actions à cette $\delta o ́ \xi \alpha$ en vue de gagner la considération de

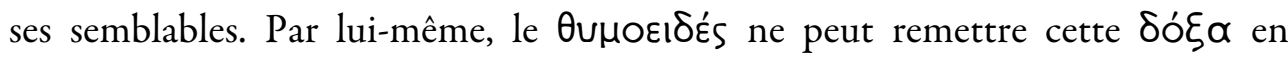
question, puisque cela supposerait qu'il raisonne, ce qui lui est impossible.

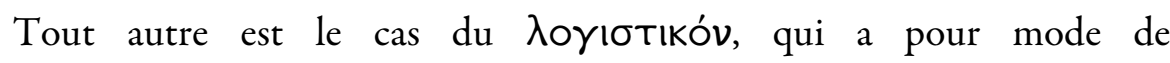

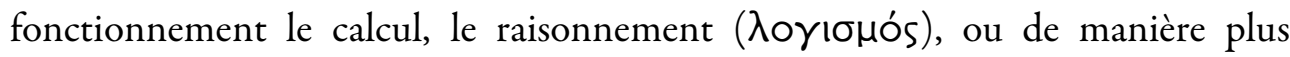
générale le $\lambda o ́ \gamma o s$ (cf. $440 \mathrm{~d} 3$ ), et pour objet de son désir « la connaissance de la

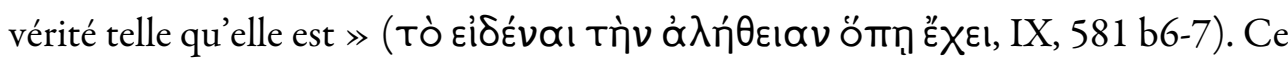

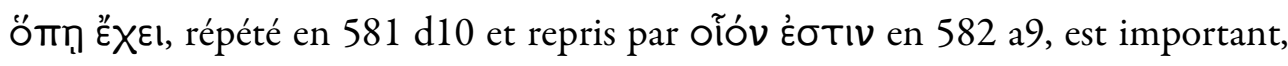

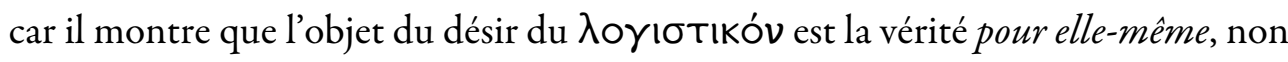
soumise à quelque autre fin. En tant que tel, tout raisonnement vise la véritée ${ }^{20}$.

\footnotetext{
${ }^{20}$ Certes, il est toujours possible d'utiliser un raisonnement pour induire les autres en erreur, par

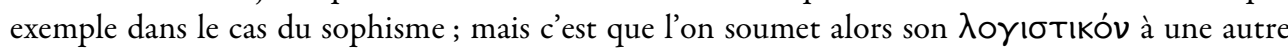

PLATO, The electronic Journal of the International Plato Society, n 8, 2008. http://gramata.univ-paris1.fr/Plato

(c) All rights of reproduction of any form reserved. 
Cependant, cela ne nous dit pas encore de quel type de vérité il s'agit, et plus loin, Socrate citera aussi bien l'opinion vraie que la science et l'intelligence parmi les

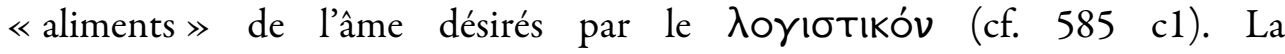
«connaissance » dont il est question dans la ligne citée ci-dessus doit donc être

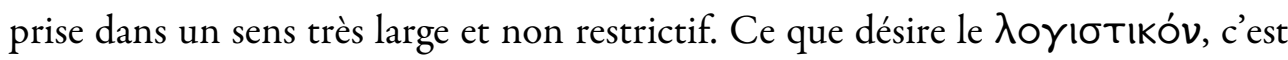
simplement la saisie de la vérité en tant que telle ; mais par lui-même, il ne sait pas encore nécessairement où chercher la vérité authentique, et même lorsqu'il le sait, cela ne l'empêche pas de désirer également la vérité qui se trouve dans les opinions. En d'autres termes, la vérité désigne seulement ici le contenu de l'objet

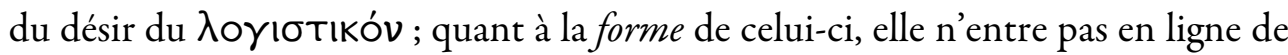
compte à ce niveau. Le sens de cette remarque s'éclairera lorsque nous tenterons de distinguer entre parties de l'âme et facultés.

\section{d) Relations entre les parties de l'âme}

Chaque partie de l'âme se compose donc d'un ensemble de mouvements psychiques ayant une même structure, c'est-à-dire un mode de fonctionnement dirigé vers un objet de désir qui lui est propre. Cette structure peut être considérée comme le principe des mouvements relevant de la partie qu'elle définit. Nous avons dès lors en nous trois principes de mouvements distincts, qui entrent souvent en conflit. En ce sens, on peut dire que nous avons « trois âmes ». Pour comprendre cette conclusion apparemment étrange, il convient de revenir sur l'analogie avec la cité. En effet, selon Socrate, une cité n'est une que lorsque la justice y règne; en revanche, dès qu'en elle s'opposent différents groupes de citoyens, elle n'est plus une seule cité, mais deux ou quelque autre nombre, qui se trouvent simplement habiter le même lieu (cf. VIII, 551 d5-7). C'est dans le même sens que l'âme de l'homme injuste n'est une qu'en apparence, mais est en

fin, en particulier le plaisir, comme les Dialogues platoniciens le montrent à l'envie (voir en particulier l'Euthydème).

PLATO, The electronic Journal of the International Plato Society, n 8, 2008. http://gramata.univ-paris1.fr/Plato

(c) All rights of reproduction of any form reserved. 
réalité multiple, puisqu'elle est traversée par des séditions internes continuelles (cf. 554 d9-e2). Cela est exprimé de manière particulièrement forte par l'image de l'âme que propose Socrate à la fin du livre IX (588 b10-e3), dont la forme

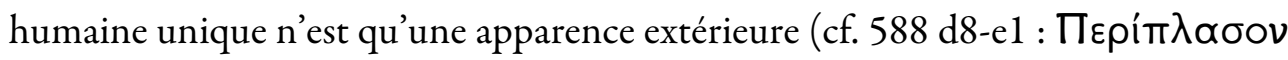

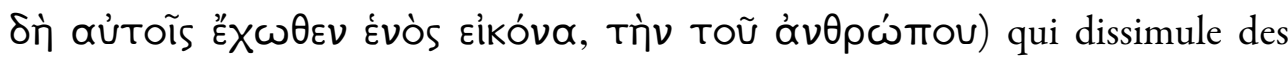

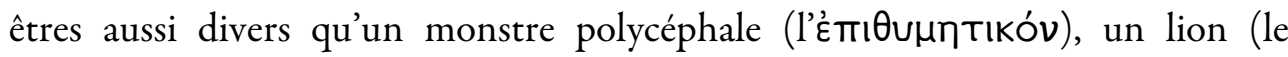

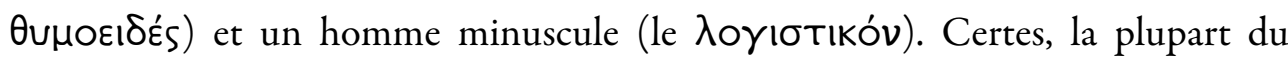
temps, l'une des trois parties prend le dessus sur les deux autres et devient ainsi le principe de direction ('ơpXń) de l'ensemble de l'âme (cf. IX, 581 c1-2). Seule cette partie a alors le pouvoir de se diriger soi-même (cf. Tทे 550 b5-6), tandis que les autres sont contraintes de lui obéir telles des esclaves - le $\lambda$ 入yı́tı́óv calculant par exemple seulement les moyens d'atteindre les fins

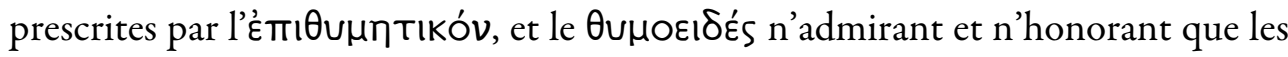
valeurs posées par celui-ci (cf. $553 \mathrm{~d} 1-7$ ). Cependant, les autres parties n'en perdent pas pour autant leur intérêt propre, qui est seulement étouffé par cette domination usurpée contre leur gré, et qui ne demande qu'à resurgir dès que l'occasion se présente. C'est pourquoi une telle âme n'est jamais à l'abri de conflits

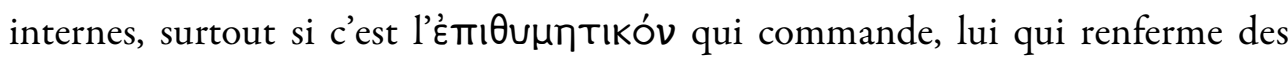
désirs variés dont les conséquences sont souvent incompatibles entre elles.

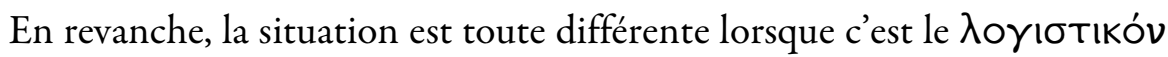
qui domine. En effet, seul celui-ci possède en lui la connaissance de ce qui est avantageux pour chacun et pour la totalité commune que constituent ces trois

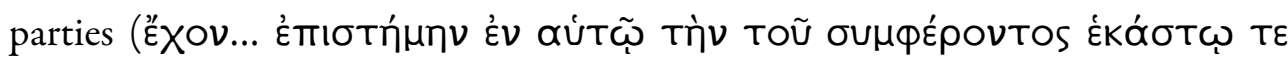

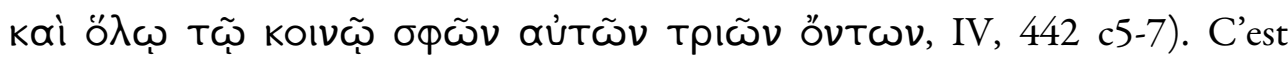
pourquoi les autres parties peuvent se soumettre de leur plein gré à cette partie, puisque cela ne les oblige aucunement à renoncer à leur propre intérêt ni à leur propre plaisir (cf. IX, 586 e4-587 a3) ; de sorte que dans ces conditions, l'accord se fait sur qui doit gouverner, ce qui correspond à la définition de la modération

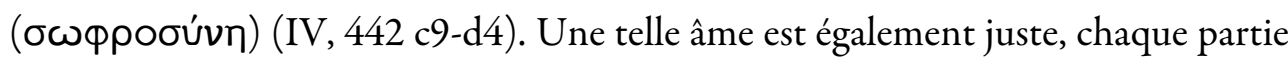
$\mathrm{y}$ accomplissant sa fonction propre et ne cherchant pas à empiéter sur les prérogatives des autres (442 d5-444 a3). Cela signifie qu'elle n'est plus tiraillée

PLATO, The electronic Journal of the International Plato Society, n 8, 2008. http://gramata.univ-paris1.fr/Plato

(c) All rights of reproduction of any form reserved. 
par des conflits internes, tous ses mouvements émanant en définitive d'un seul et

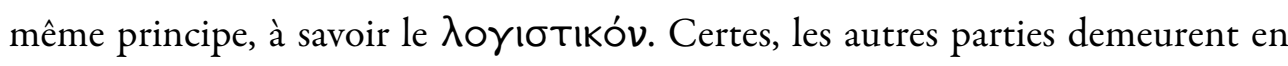
droit des principes de mouvements, puisque telle est leur nature intime ; c'est

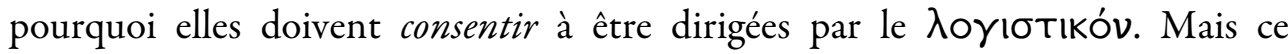
faisant, elles abandonnent de leur plein gré l'usage de leur capacité à se diriger soi-

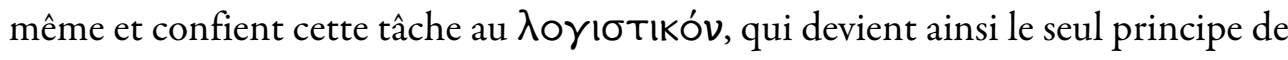
mouvement réellement agissant dans cette âme.

Remarquons que cet accord entre les parties de l'âme de l'homme juste implique que dans son cas, le principe qui nous a permis d'établir la tripartition de l'âme ne fonctionnera plus, car l'observation empirique ne nous révélera aucun conflit en lui. En ce sens, l'âme juste n'est plus « trois », mais « une » - ce qui ne veut pas dire qu'il n'y a en elle aucune multiplicité, puisque par définition elle est principe d'une multiplicité de mouvements psychiques, et même de mouvements psychiques d'espèces différentes, mais seulement que ces mouvements sont « harmonisés » de telle manière qu'ils tendent tous dans la même direction, l'homme juste devenant ainsi tout à fait un à partir de plusieurs ( $\pi \propto v$ Tá $\pi \propto \sigma ı v$

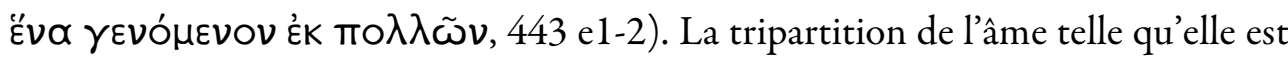
examinée dans le livre IV n'appartient donc pas à l'essence de l'âme, mais correspond plutôt à un certain état de l'âme humaine, à savoir l'injustice. C'est sans doute pour cette raison que Socrate semble émettre quelques doutes sur cette tripartition au livre X (cf. 611 a10-612 a7): tout dépend en effet de l'âme envisagée, l'argument du livre IV en faveur de la tripartition n'étant valable que pour l'âme injuste. Cela ne signifie pas que les trois parties ne demeurent pas en droit distinguables chez le juste également, puisque même lui doit bien avoir des désirs tels que la soif ou la faim, et s'emportera sans doute également lorsque la justice lui paraîtra violée ; mais tous ces élans se dirigeront vers le même but, tels les chevaux et le cocher de l'attelage ailé représentant l'âme des dieux dans le Phèdre (246 a7-8, 247 b1-2).

PLATO, The electronic Journal of the International Plato Society, n 8, 2008. http://gramata.univ-paris1.fr/Plato

(c) All rights of reproduction of any form reserved. 
III. Les facultés

\section{a) Définition et principe de distinction des facultés}

L'étude des facultés intervient dans un tout autre contexte, à savoir la distinction entre philodoxe et philosophe effectuée à la fin du livre $\mathrm{V}$ de la République (475 d1-480 a13). Afin d'établir cette distinction, Socrate cherche à

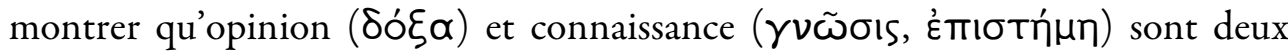

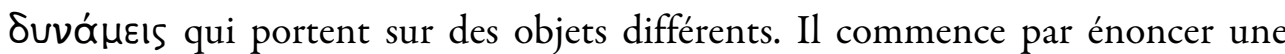

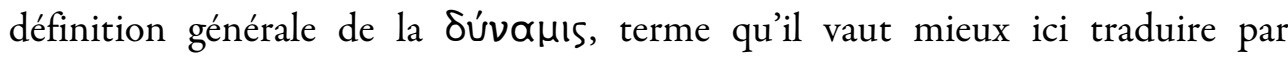
« capacité », étant donné l'extension extrêmement large que lui confère Socrate : « Nous affirmerons que les capacités sont un certain genre d'êtres, par lesquelles nous en particulier pouvons ce que nous pouvons et toute autre chose peut ce que

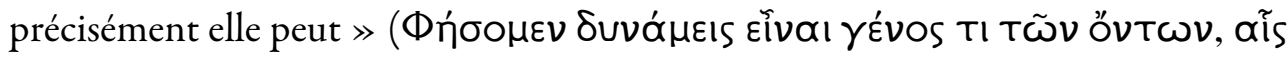

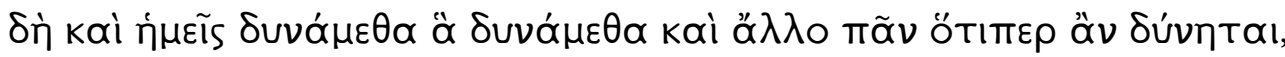
477 c1-2). Ainsi, la $\delta$ ó $\alpha$ est une capacité, puisqu'elle n'est rien d'autre que « ce

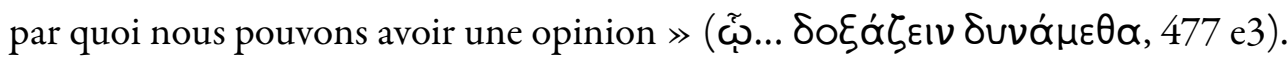
De même, la connaissance est une capacité ( $477 \mathrm{~d} 8$-e1), à savoir celle par laquelle

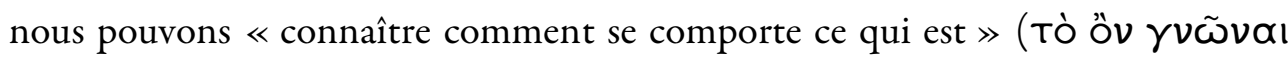

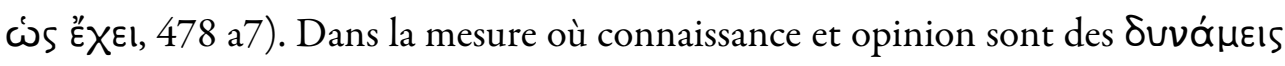
d'ordre cognitif, il semble permis d'adopter dans leur cas la traduction traditionnelle de ce terme par « facultés ${ }^{21}$.

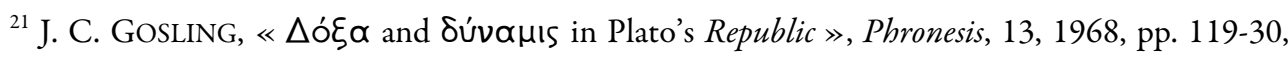
s'oppose à cette traduction, considérant que des passages tels que République, VII, 518 b7-519 a6,

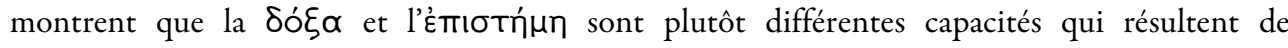
l'application de la même faculté (pp. 128-9). Tout dépend bien entendu de ce que l'on entend par le terme « faculté », l'usage qu'en fait Gosling s'appliquant en réalité ici à la partie rationnelle de l'âme, comme je le montrerai plus loin. Un tel usage me paraît particulièrement problématique chez Platon, en ce qu'il ne peut qu'entretenir la confusion entre partie de l'âme et faculté, confusion sur laquelle je reviendrai. Puisque c'est ce texte du livre V de la République qui introduit

PLATO, The electronic Journal of the International Plato Society, n 8, 2008.

http://gramata.univ-paris1.fr/Plato

(c) All rights of reproduction of any form reserved. 
Socrate énonce ensuite un critère permettant de distinguer les capacités en général :

Dans une capacité, moi, je ne vois ni une quelconque couleur, ni une quelconque figure, ni aucune autre qualité de ce genre, comme c'est le cas pour de nombreuses autres choses, qualités relativement auxquelles, en tournant mon regard vers elles, je distingue certaines choses pour moi-même en disant qu'elles sont différentes les unes des autres. Dans une

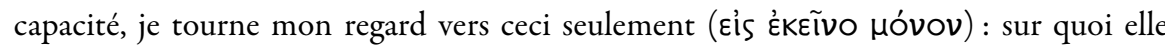

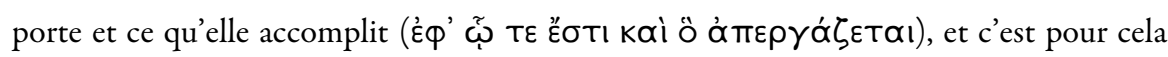
que j'appelle chacune d'entre elles une capacité, et celle qui est assignée à la même chose

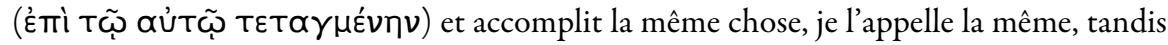
que celle qui est assignée à autre chose et accomplit autre chose, je l'appelle autre. (477 c6-d5)

Socrate ne semble pas envisager «ce sur quoi elle porte» et «ce qu'elle accomplit » comme deux critères distincts, sans quoi il oublierait de mentionner

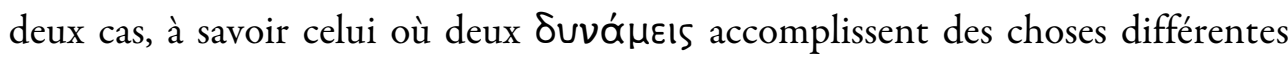
relativement au même objet, et celui où deux Suváukıs accomplissent la même chose relativement à des objets différents. Or l'exclusion de ces deux possibilités est cruciale pour la validité du raisonnement qui suit, qui va établir que la $\delta o ́ \xi \alpha$ et la connaissance, parce qu'elles accomplissent des choses différentes, ont nécessairement des objets différents. Il faut donc interpréter ces deux aspects comme les deux versants inséparables d'un seul et même critère, c'est-à-dire non pas nécessairement comme impliquant une identité entre ce qu'accomplit une

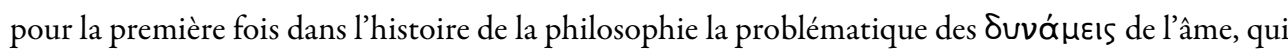
deviendra par la suite celle des facultés, il me semble nettement préférable d'adopter cette traduction traditionnelle, tout en prenant garde de ne pas importer par la même occasion les conceptions que les philosophes ultérieurs se feront de ces facultés. - Pour une définition « classique » du terme «faculté », cf. A. LALANDE, Vocabulaire technique et critique de la philosophie, Paris, 1926 (réédition aux P.U.F. dans la collection «Quadrige », 1993, T. I, p. 333), s.v., sens B : « on appelle Facultés de l'âme l'intelligence, l'activité (ou, comme on disait autrefois, la volonté), et enfin la sensibilité, en tant qu'elles sont considérées comme constituant chacune un pouvoir spécial de faire ou de subir un certain genre d'action » (souligné dans le texte).

PLATO, The electronic Journal of the International Plato Society, n 8, 2008.

http://gramata.univ-paris1.fr/Plato

(c) All rights of reproduction of any form reserved. 
capacité et son objet ${ }^{22}$, mais du moins de telle manière qu'une capacité ne puisse accomplir ce qu'elle accomplit que parce qu'elle porte sur tel objet, et qu'elle ne puisse porter sur cet objet que parce qu'elle accomplit ce qu'elle accomplit ${ }^{23}$.

\section{b) Connaissance et opinion}

Résumons le raisonnement de Socrate. Il commence par faire reconnaître

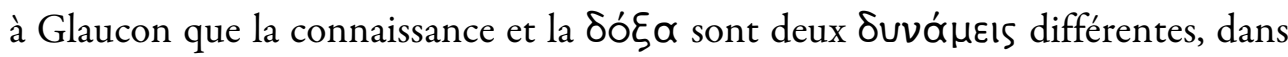

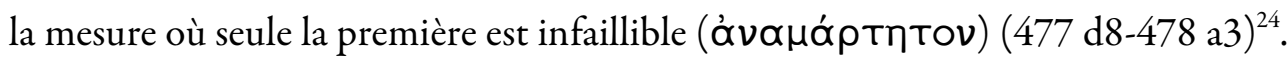

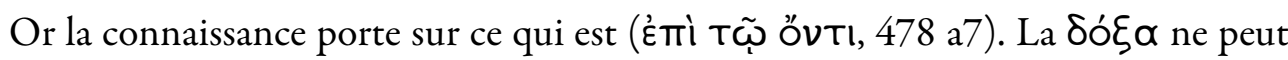
donc pas porter sur ce qui est, puisqu'elle doit avoir un objet différent de la connaissance (478 a11-b4). Elle ne peut toutefois pas non plus porter sur ce qui n'est pas, car elle porte bien sur quelque chose, seule l'ignorance pouvant être rapportée à ce qui n'est pas, c'est-à-dire à ce qui n'est rien (478 b5-c6). Intermédiaire entre l'ignorance et la connaissance, la $\delta o ́ \xi \alpha$ doit porter sur un objet intermédiaire entre ce qui est et ce qui n'est pas, à savoir quelque chose qui à

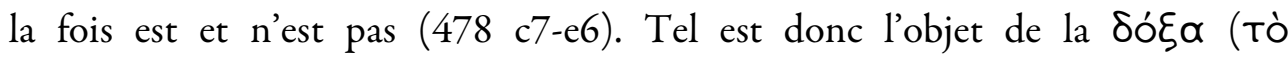

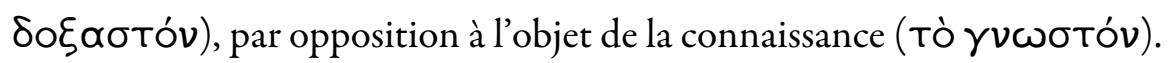

${ }^{22}$ Comme l'écrit J. HiNTIKKA, « Knowledge and its objects in Plato », dans J. M. E. MORAVCSIK (éd.), Patterns in Plato's Thought, Dordrecht/Boston, 1973, pp. 12-16, dont la position est critiquée par G. SANTAS, «Hintikka on Knowledge and its objects in Plato », dans le même recueil, pp. 36-42.

${ }^{23}$ Voir notamment G. SANTAS, art. cit., pp. 44 sq. ; J. ANNAS, An Introduction to Plato's Republic, Oxford, 1981, p. 202 ; N. COOPER, « Between knowledge and ignorance », Phronesis, 31, 1986, p. 231 ; L. P. Gerson, Knowing Persons. A Study in Plato, Oxford, 2003, pp. 155-6; T. A. SZLEZÁK, Die Idee des Guten in Platons Politeia. Beobachtungen zu den mittleren Büchern, Sankt Augustin, 2003, p. 59. Comme le remarque N. Cooper - à la suite de J. HINTIKKA, art. cit., p. 15

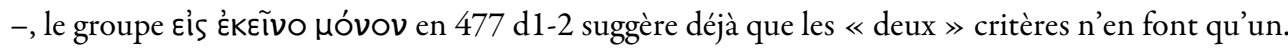
Le fait qu'ils soient reliés par Tع... kơi peut d'ailleurs également plaider en ce sens, puisque dans les cas de ce genre, «le second membre sert souvent à expliquer le premier » (R. KÜHNER et B. GERTH, Ausführliche Grammatik der Griechischen Sprache [1898], Zweiter Teil: Satzlehre, Hannovre/Leipzig, $1904^{3}$, Vol. II, $\$ 522$, p. 249).

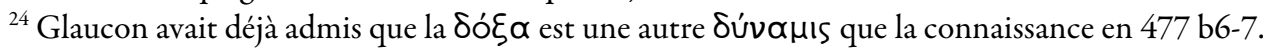

PLATO, The electronic Journal of the International Plato Society, n 8, 2008.

http://gramata.univ-paris1.fr/Plato

(c) All rights of reproduction of any form reserved. 
La difficulté principale de ce raisonnement tient à la compréhension de sa prémisse, posée comme évidente, selon laquelle seul ce qui est peut être objet de connaissance, tandis que relativement à ce qui n'est pas, seule l'ignorance est possible (cf. 476 e7-477 a5). Et que dire de cette proposition étrange selon laquelle la $\delta o ́ \xi \alpha$ porte sur ce qui à la fois est et n’est pas, « intermédiaire » entre l'être et le non-être! Heureusement, Socrate nous explique ensuite ce qu'il faut entendre par là : les objets de la $\delta o ́ \xi \alpha$ ne sont, pas plus qu'ils ne sont pas, ce que l'on dit qu'ils sont - par exemple, une chose dite belle est également laide sous quelque aspect, de même qu'une chose juste est également injuste, une chose pieuse, impie, et une chose deux fois plus grande, deux fois moins grande (478 e7479 d5). Parce qu'ils peuvent être considérés sous différents rapports, à différents moments et dans différentes relations, les objets de la $\delta o ́ \xi \alpha$ ne sont jamais purement et simplement ce qu'ils sont, mais admettent toujours également les prédicats contraires, qui impliquent la négation des prédicats qui leur étaient tout d'abord attribués. Dans ces conditions, si l'objet de la connaissance s'identifie quant à lui à ce qui est, il doit être purement et simplement ce qu'il est, et jamais le contraire, quelle que soit la manière dont on l'envisage : il doit être «toujours

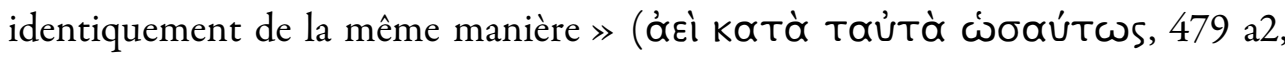
e6-7).

On voit que le verbe « être » doit ici être pris non en un sens existentiel, mais au sens où être, c'est toujours être quelque chose, c'est-à-dire être déterminé d'une certaine manière ${ }^{25}$. En ce sens, ce qui n'est absolument pas ( $\mu$ ì őv... $\mu \eta \delta \propto \mu \tilde{n}, 477$ a3), c'est ce qui est totalement indéterminé, relativement auquel seule l'ignorance la plus complète est possible, puisque tant la $\delta o ́ \xi \alpha$ que la connaissance sont par nature des activités déterminantes. L'objet d'opinion, lui, est bien déterminé sous un certain rapport, mais justement, il ne peut l'être que sous un certain rapport, parce qu'il appartient à sa nature de pouvoir être

\footnotetext{
${ }^{25}$ Sur cette compréhension de l'être, que je ne peux ici qu'effleurer, je me permets de renvoyer à mes contributions au volume collectif dirigé par A. MOTTE et P. SOMVILLE, OUSIA dans la philosophie grecque des origines à Aristote, Travaux du Centre d'études aristotéliciennes de l'Université de Liège, Louvain-la-Neuve, Peeters, 2008, pp. 101-11 (sur la République) et 131-42.
}

PLATO, The electronic Journal of the International Plato Society, n 8, 2008. http://gramata.univ-paris1.fr/Plato

(c) All rights of reproduction of any form reserved. 
considéré sous de multiples perspectives différentes qu'il est impossible de rassembler en un point de vue unique tant que l'on en reste au niveau du sensible. C’est pourquoi la $\delta o ́ \xi \alpha$ n'est pas infaillible: toute $\delta o ́ \xi \alpha$, même vraie, est également fausse d'une certaine manière, parce qu'elle confère indûment une valeur universelle à ce qui n'est vrai que dans une perspective particulière et ponctuelle. En revanche, l'objet de la connaissance est déterminé sous tous les rapports, de sorte qu'il reste identique à lui-même lorsqu'on le confronte à d'autres objets du même type. Un tel objet parfaitement déterminé en lui-même est ce que Platon nomme une Idée; et la connaissance, en tant qu'elle est saisie de cette Idée et de la manière dont elle se comporte, est nécessairement infaillible et toujours vraie.

Cette interprétation semble s'imposer comme étant à la fois la plus naturelle et la plus conforme au texte. Elle permet par ailleurs de comprendre en


n'en font qu'un, c'est-à-dire en quel sens ce qu'accomplit chaque capacité est nécessairement lié à la nature de ce sur quoi elle porte, et inversement. Car non seulement les objets en question ne peuvent être saisis que par les facultés qui leur sont assignées, mais inversement ces facultés ne peuvent s'exercer que sur ces objets: l'ignorance n'est possible que relativement à ce qui est absolument indéterminé $^{26}$, la $\delta o ́ \xi \alpha$, relativement à ce qui n'est que partiellement déterminé27, et la connaissance au sens propre, relativement à ce qui est pleinement déterminé.

\footnotetext{
${ }^{26}$ Rappelons que l'ignorance n'est pas ici l'ignorance empirique de tel ou tel individu, mais

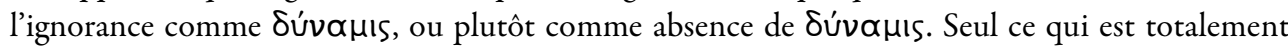
indéterminé, et en ce sens n'est rien, ne peut être saisi de quelque manière que ce soit.

${ }^{27}$ Que Socrate dise plus loin avoir une Sóga relativement à l'Idée du bien (VI, 506 c6) ne contredit pas cette conclusion, contrairement à ce qu'écrit G. FINE, « Knowledge and belief in Republic V » [1978], repris dans Plato on Knowledge and Forms, Oxford, 2003, p. 66, car il est clair qu'il ne suffit pas de nommer une Idée quelconque pour la connaître effectivement. Il est possible d'avoir une $\delta o ́ \xi \alpha$ relative à l'Idée du bien, mais dans ce cas on ne la saisit précisément pas dans sa pleine déterminité, c'est-à-dire en tant qu'Idée : on l'entrevoit seulement obscurément, comme étant partiellement déterminé, c'est-à-dire en tant qu'objet d'opinion.
}

PLATO, The electronic Journal of the International Plato Society, n 8, 2008.

http://gramata.univ-paris1.fr/Plato

(c) All rights of reproduction of any form reserved. 


\section{c) Nature de la relation entre une faculté et son objet}

On a pourtant parfois tenté de résister à cette interprétation, sous prétexte qu'elle impliquerait une « théorie des deux mondes » par trop absurde et indéfendable ${ }^{28}$. Mais une telle objection me semble reposer sur une compréhension erronée de ce qu'il faut entendre par l' «objet » d'une faculté.

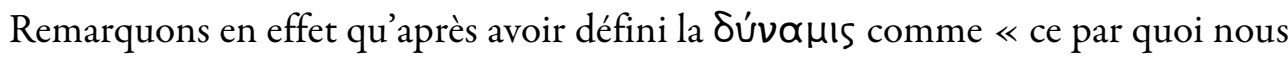
pouvons ce que nous pouvons », Socrate cite comme exemples de Suváukıs la vue (őభıs) et l'ouie (ákoń) (V, 477 c3). Il est vrai qu'il ne précise pas ici l'objet sur lequel porte chacune de ces $\delta$ uvá $\mu \varepsilon \mathrm{s}^{29}$; mais il le fait au livre VI, dans le cadre de l'analogie entre l'Idée du bien et le soleil, en déclarant que par la vue (

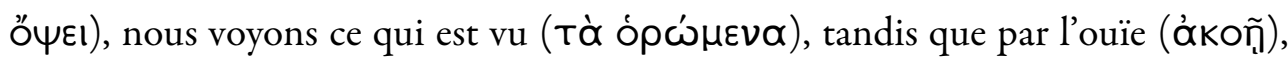
nous entendons ce qui est entendu (Tà ớkovó $\mu \varepsilon v \alpha$ ), et par les autres sensations

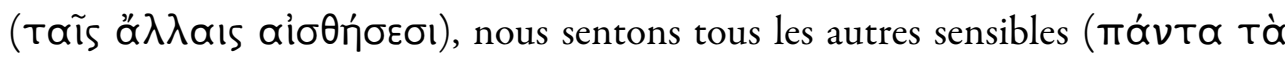

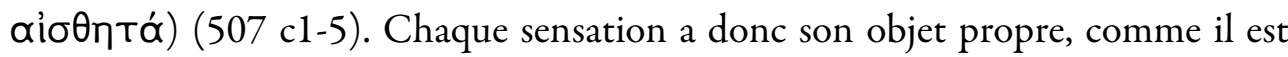

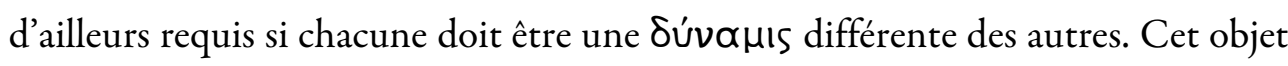
n'est pas la chose sensible en général, ni même la chose sensible envisagée sous tel ou tel aspect - l'aspect par lequel elle est visible, audible, etc. $-^{30}$, mais purement et simplement cet aspect lui-même, considéré en lui-même et non comme l'aspect

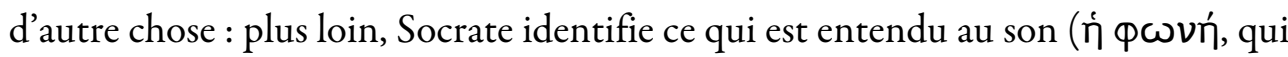
doit ici avoir ce sens très général), et le visible (ici tò óparóv) à la couleur (ín

${ }^{28}$ Cf. J. C. GosLing, art. cit., p. 124; G. FINE, art. cit., p. 66. Pour une critique radicale (et parfaitement fondée à mes yeux) de l'interprétation de Fine, cf. F. J. GONZALEZ, « Propositions or objects ? A critique of Gail Fine on knowledge and belief in Republic V », Phronesis, 41, 1996, pp. 245-75. On trouve également une défense récente de la connexion de cette distinction entre

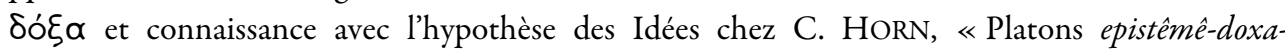
Unterscheidung und die Ideentheorie (Buch V 474 b-480 a und Buch X 595 c-597 e) », dans O. Höffe (éd.), Platon : Politeia, Berlin, 1997, pp. 291-312 et chez L. P. GERSON, op. cit., pp. 14873.

${ }^{29}$ Comme le remarque J.C. GOSLING, art. cit., p. 123.

${ }^{30}$ En ce sens, mon interprétation differe de celle de N. D. SMITH, «Republic 476 e-480 a: intensionality in Plato's epistemology? », Philosophical Studies, 30, 1976, pp. 427-9, qui a toutefois le mérite de voir le problème que pose la lecture traditionnelle de ce passage.

PLATO, The electronic Journal of the International Plato Society, n 8, 2008.

http://gramata.univ-paris1.fr/Plato

(c) All rights of reproduction of any form reserved. 


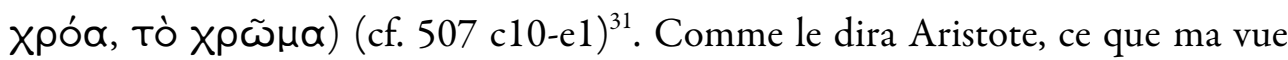
voit en propre, ce n'est pas le fils de Diarès, mais le blanc, qui se trouve être le fils de Diarès par accident (De Anima, II, 6, 418 a20-23 ; voir aussi III, 1, 425 a25-

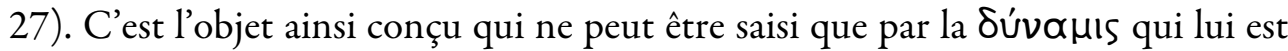
assignée, tandis que la «chose » colorée ou sonore peut très bien également être

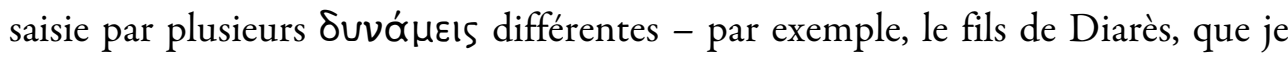
peux non seulement voir, mais encore entendre, toucher, à propos duquel je peux avoir des $\delta o ́ \xi \alpha ı$, etc. On comprend dès lors pourquoi chaque faculté est « par

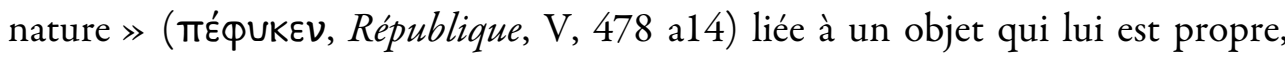
puisque celui-ci est lui-même fonction de la faculté qui l'appréhende. En ce sens, la relation entre une faculté et son objet peut être rapprochée de la relation noético-noématique husserlienne, dont il ne me paraît pas exagéré de repérer l'acte de naissance dans ce passage de la République ${ }^{32}$. Encore une fois, il ne s'agit pas simplement de dire que différentes facultés envisagent le même objet sous des rapports différents, mais bien que leurs objets eux-mêmes sont différents, car ces objets ne sont rien d'autre que ce que la faculté en saisit, leur pur et simple « apparaître » à la faculté en question. Que cet apparaître puisse être considéré par ailleurs comme un apparaître « de » quelque chose d'autre, qui se cacherait « sous » cette apparence, n'a aucune pertinence eu égard à ce problème. En ce sens, dire que la $\delta o ́ \xi \alpha$ et la connaissance ont des objets différents signifie simplement que la nature de ce que ces deux facultés saisissent est essentiellement différente, mais nullement qu'à ces objets correspondraient des «choses » appartenant à deux « mondes » distincts; et parler d'un « monde des Idées » en l'opposant à un « monde sensible » n'a ni plus ni moins de sens que parler d'un «monde des couleurs» ou d'un «monde des sons», expressions qui ne désignent certes pas différents plans de réalités qui se superposeraient les uns aux autres, mais seulement les domaines des objets des facultés correspondantes.

\footnotetext{
${ }^{31}$ Voir d'ailleurs déjà Charmide, $168 \mathrm{~d} 1$-e2.

${ }^{32}$ Voir notamment E. HUSSERL, Idées directrices pour une phénoménologie, Trad. P. RICOEUR, Paris, 1950, \$\$87-96.
}

PLATO, The electronic Journal of the International Plato Society, n 8, 2008. http://gramata.univ-paris1.fr/Plato

(c) All rights of reproduction of any form reserved. 
Cette liaison entre une faculté et son objet doit être distinguée de celle que nous avons repérée plus haut entre une partie de l'âme et l'objet de son désir. Tout d'abord, elle est exclusive : une faculté ne peut entrer en relation qu'avec son objet propre, et cet objet ne peut être saisi que par cette faculté. En revanche, nous l'avons vu, le fait que chaque partie de l'âme ait son propre objet de désir ne signifie pas qu'elle ne puisse entrer en relation qu'avec cet objet, ni que cet objet ne puisse être saisi que par cette partie de l'âme, puisque tout le raisonnement aboutissant à distinguer les parties de l'âme repose sur la possibilité pour ces parties d'entrer en relation avec un même objet (mpòs taủtóv). Par ailleurs, l'objet d'une faculté se définit exclusivement par sa forme, c'est-à-dire par ce qui en fait tel type d'être, et non par son contenu : l'objet de la vue doit être visible,

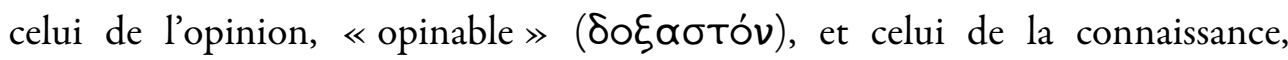
connaissable. Une telle exigence n'a aucune implication sur le contenu du type d'être ainsi défini : il est clair que nous n'avons pas besoin de facultés différentes pour saisir le rouge, le vert et le jaune. Bien plus, il est tout à fait possible que le contenu des objets de différentes facultés soit le même; par exemple, le rouge peut être appréhendé tantôt par la vue, tantôt par l'opinion, tantôt par la connaissance. Certes, ce n'est pas du même rouge qu'il s'agit dans les trois cas, mais les différences tiennent ici à la forme qu'il revêt en relation avec telle faculté particulière, et non à son contenu: si le rouge comme objet d'opinion est différent du rouge comme objet de connaissance, c'est parce que seul le second est une Idée, et est dès lors seulement rouge, tandis que le premier est tout autant rouge que non rouge sous certains rapports. En revanche, l'objet du désir de chacune des parties de l'âme est un simple contenu (le plaisir, l'honneur, la vérité), qui ne nous renseigne encore aucunement sur la forme que doit revêtir ce

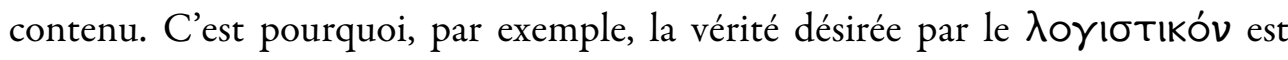
tout autant celle de la $\delta o ́ \xi \alpha$ que celle de la connaissance. Ces deux différences entre la liaison d'une faculté à son objet et celle d'une partie de l'âme à son objet de désir ne sont d'ailleurs pas indépendantes l'une de l'autre : c'est parce que les parties de l'âme ne sont pas liées par nature à une forme d'objet particulière qu'il

PLATO, The electronic Journal of the International Plato Society, n 8, 2008. http://gramata.univ-paris1.fr/Plato

(c) All rights of reproduction of any form reserved. 
est possible à l'une d'entrer en relation avec l'objet du désir de l'autre, tandis que cela est absolument impossible dans le cas des facultés, puisque la forme de l'objet varie en raison directe des variations de la faculté qui le saisit. Cela nous montre déjà qu'il faut absolument éviter d'identifier parties de l'âme et facultés chez Platon - identification que celui-ci n'effectue jamais dans les textes, mais à laquelle nous sommes naturellement portés en raison de l'influence d'Aristote ${ }^{33}$.

\section{d) La sensation}

Revenons à présent au cas des sensations. Le fait que les différentes sensations aient chacune leur objet propre n'interdit pas pour autant de parler du sensible en général; simplement, celui-ci n'est pas l'objet d'une sensation particulière, ni même de toutes les sensations particulières, mais de la sensation en tant que telle, considérée dans sa généralité. Ceci résulte nécessairement du principe de détermination réciproque des corrélatifs que nous avons rencontré lorsque nous étudiions la problématique des parties de l'âme. Cependant, la

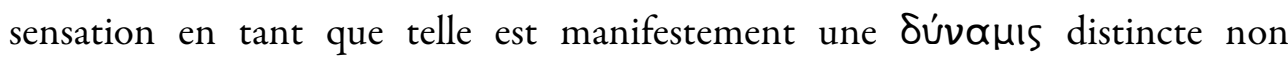
seulement de la connaissance, mais également de la $\delta o ́ \xi \alpha$. Son objet, le sensible, doit donc être différent de celui de la $\delta o ́ \xi \alpha$. Pourtant, à la fin du livre VI de la

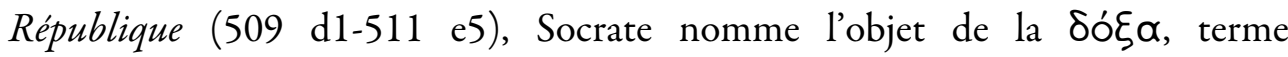
rassemblant les deux degrés inférieurs de la ligne, aussi bien «ce qui est vu » (Tò

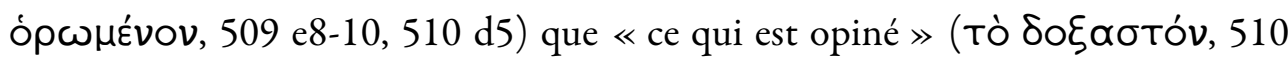
a). Bien plus, si vraiment les objets de la sensation different de ceux de la $\delta o ́ \xi \alpha$, où les placer sur l'échelle de l'être et du non-être ? Ils ne peuvent correspondre ni à ce qui est, sans quoi la sensation serait identique à la connaissance, ni à ce qui est

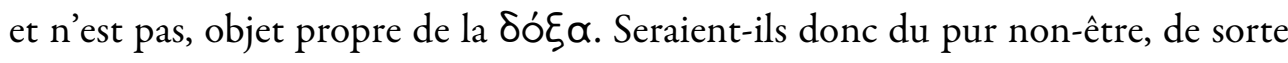
que la sensation serait tout bonnement ignorance ? Un bref détour par un passage

${ }^{33}$ Pour un exemple particulièrement clair de confusion entre parties de l'âme et facultés, cf. A. GRAESER, Probleme der platonischen Seelenteilungslehre, München, 1969. Voir en revanche l'excellente mise au point de J. MOLINE, art. cit., pp. 1-26.

PLATO, The electronic Journal of the International Plato Society, n 8, 2008. http://gramata.univ-paris1.fr/Plato

(c) All rights of reproduction of any form reserved. 
capital du Théétète (184 b3-187 a9) va nous permettre de répondre à ces questions.

Ce passage intervient à la fin de l'examen de la définition de la science comme sensation, dont il constitue la réfutation définitive. Socrate commence

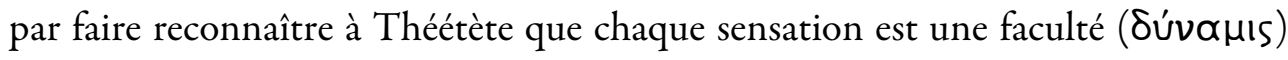
qui a son objet propre - par exemple le son ( $\phi \omega \nu \eta ́)$ pour l'ouie et la couleur (Xpóa) pour la vue (185 a8) -, ce qui peut être senti par l'une ne pouvant l'être par l'autre, et inversement (184 e8-185 a3). En cela, le Théétète ne fait que confirmer ce que nous avons lu dans la République. Mais Socrate en tire une

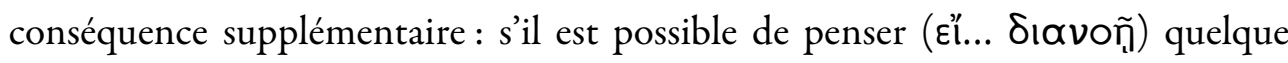
chose qui appartienne à deux de ces objets à la fois, par exemple au son et à la couleur, ce quelque chose ne peut être senti ni par le canal de l'œil, ni par le canal de l'oreille (185 a4-7). Or l'une des choses qu'il est possible de penser à propos de la couleur et du son, c'est que tous les deux sont (185 a8-10). Une telle détermination, comme celles de l'altérité, de l'identité, du nombre, etc., ne peut nous venir de la sensation, mais doit être le fait de l'âme elle-même (185 c4-186 a1). La sensation en tant que telle n'atteint donc pas l'être (186 e1-6). Cela ne signifie pas pour autant qu'elle atteint le non-être, car celui-ci appartient tout autant aux déterminations « communes » qui sont le fait de l'âme elle-même (cf. 185 c9) ; mais il faut bien plutôt comprendre que l'objet de la sensation est en deçà de l'être et du non-être, la sensation ne pouvant saisir de telles déterminations. C'est pourquoi il ne faut pas chercher à situer cet objet dans la gradation proposée à la fin du livre $\mathrm{V}$ de la République entre ce qui n'est absolument pas, ce qui est et n'est pas et ce qui est absolument, car il n'a pas à y figurer. En revanche, la $\delta o ́ \xi \alpha$ atteint bien quant à elle le plan de l'être et du nonêtre, puisque cet acte par lequel l'âme examine les déterminations communes des

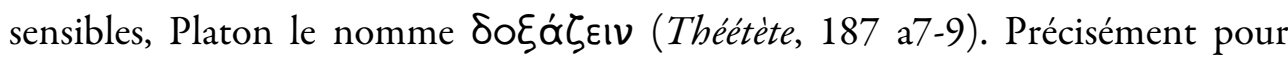
cette raison, l'objet de la $\delta o ́ \xi \alpha$, même lorsqu'il s'agit d'une chose sensible, est différent de celui de la sensation brute : il est déjà «structuré » par une $\delta o ́ \xi \alpha$ qui en fait un objet déterminé, qui est, est un, identique à lui-même en tant

PLATO, The electronic Journal of the International Plato Society, n 8, 2008. http://gramata.univ-paris1.fr/Plato

(c) All rights of reproduction of any form reserved. 
qu'objet d'une opinion etc. Cet objet, c'est ce que Platon nomme la $\phi \alpha v \tau \alpha \sigma i ́ \alpha$, ou encore le paívetaı et le paıvó $\mu \varepsilon v o v$, et qu'il définit dans le Sophiste comme

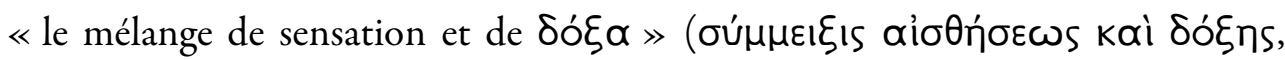
264 b2). Mais puisqu'un tel objet contient à la fois une sensation et une $\delta o ́ \xi \alpha$, on peut aussi bien le désigner tantôt par l'un, tantôt par l'autre terme, et c'est ce que fait Socrate à la fin du livre VI de la République.

\section{e) Faculté et organe}

Le texte du Théétète qui vient d'être mentionné nous permet également d'introduire une distinction supplémentaire: celle entre organe et faculté,

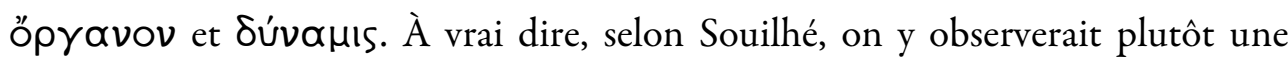
confusion entre ces deux termes ${ }^{34}$; mais rien dans le texte ne me paraît légitimer cette conclusion, qui nous forcerait à reconnaître que Platon confond l'œil et la vue. Un simple retour à la République suffira à montrer que tel n'est nullement le cas, puisque dans ce dialogue, Socrate distingue très clairement la vue de l'œil (Tò

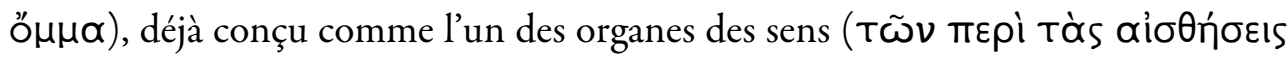

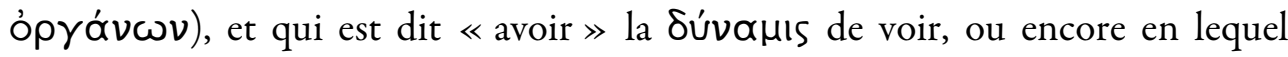
celle-ci est dite «se trouver » (cf. VI, 508 a11-b8). D'ailleurs, le simple fait que les organes soient corporels, à la différence des facultés qui n'ont ni couleur, ni figure, ni aucune autre qualité de ce genre, suffit à les distinguer. Mais les facultés

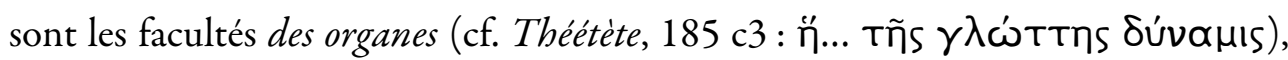
et c'est pourquoi on peut identifier «ce au moyen de quoi » $\left(\delta \iota^{\prime}\right.$ oũ) nous sentons aussi bien à l'organe (cf. 184 c5-9, d8, 185 a4-7) qu'à la faculté de cet organe (cf. 184 e8-185 a3, 185 b7-c3, 185 e7). Remarquons que dans ces conditions, les sensations ne doivent pas être considérées comme des facultés de l'âme, mais bien comme des facultés du corps, le corps étant l'organe de la

\footnotetext{
${ }^{34}$ Cf. J. SoullHÉ, Étude sur le terme $\triangle Y N A M I \Sigma$ dans les Dialogues de Platon, Paris, 1919, pp.
} 164-6.

PLATO, The electronic Journal of the International Plato Society, n 8, 2008. 
sensation en général (cf. 184 e5-7, 185 e7, 186 c1-2). Cela ne signifie pas du tout que l'âme n'intervient pas dans la sensation : au contraire, Platon est le premier à reconnaître que la sensation se fait «au moyen » du corps, mais «par » l'âme (cf. 185 c5-d6). Mais il faut distinguer la faculté, l'organe porteur de cette faculté et ce qui utilise cette faculté pour saisir l'objet qui lui correspond. L'âme utilise le corps pour saisir les différents sensibles grâce aux facultés des organes sensoriels dont celui-ci dispose.

Quant à la $\delta o ́ \xi \alpha$, elle n'a pas d'organe corporel ; mais c'est l'âme elle-

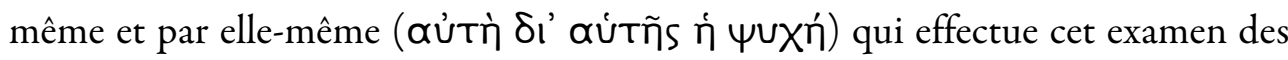
déterminations communes ( $185 \mathrm{~d} 6$-e2). Autrement dit, l'âme est à elle-même son propre « organe » relativement à la $\delta o ́ \xi \alpha$ - et aussi bien à la connaissance -, et c'est pourquoi seules celles-ci peuvent être nommées à proprement parler des facultés psychiques. Mais cet « organe » est-il l'âme dans sa totalité, ou seulement certaines de ses « parties » ? C'est ce qu'il nous reste à examiner.

\section{Rapports entre parties et facultés}

\section{a) Le problème}

On peut toutefois se demander si cette question a un sens. En effet, la problématique des parties de l'âme et celle des facultés n'interviennent-elles pas dans deux contextes tout à fait différents, l'un à connotation plutôt « éthique », l'autre à connotation « épistémologique » ? Dans ces conditions, ne faisons-nous pas fausse route en cherchant à les mettre en rapport ? À cette objection, on peut tout d'abord répondre que de telles distinctions sont toujours périlleuses relativement à Platon : par exemple, le contexte de la distinction entre les facultés a lui aussi des connotations «éthiques », puisqu'il s'agit de distinguer le philosophe du philodoxe afin de montrer que seul le premier est apte à gouverner. Quoi qu'il en soit, le fait que ces deux problématiques soient a priori

PLATO, The electronic Journal of the International Plato Society, n 8, 2008. http://gramata.univ-paris1.fr/Plato

(c) All rights of reproduction of any form reserved. 
indépendantes l'une de l'autre n'implique pas qu'elles soient incompatibles, et de toute façon, nous allons voir que Platon lui-même les met en rapport dans certains passages de la République.

Mais s'il est vrai que ce problème n'est pas en soi absurde, que signifie-t-il exactement ? Pour le comprendre, il convient de commencer par le reformuler en le « déréifiant » autant que possible. En effet, les parties de l'âme ne sont pas des parties d'un corps, à la manière des organes corporels, mais des structures qui définissent des espèces de mouvements psychiques, une structure comportant essentiellement deux aspects : un mode de fonctionnement spécifique et un objet de désir qui lui est propre. Quant aux facultés, du moins ces facultés psychiques que sont la connaissance et la $\delta o ́ \xi \alpha$, elles sont elles aussi des mouvements psychiques, ou plutôt la capacité d'effectuer un certain type de mouvements psychiques. Mais ces mouvements se définissent moins par leur structure que par leur résultat, par ce qu'ils accomplissent, à savoir la saisie de l'objet qui leur est assigné en propre. La question de savoir si les facultés relèvent de telle ou telle partie de l'âme devient alors: leur résultat ne présuppose-t-il pas lui aussi une certaine structure, c'est-à-dire un certain mode de fonctionnement et une certaine orientation qui seuls permettent de l'atteindre ? Bien que cette question ne soit jamais abordée directement par Platon, on peut en déduire la réponse de ce qui est dit en plusieurs endroits de la République.

\section{b) L'opinion}

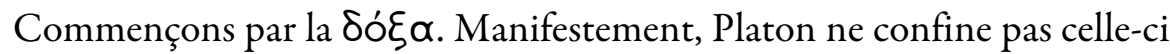
à une seule partie de l'âme : selon la République, les trois parties peuvent avoir des

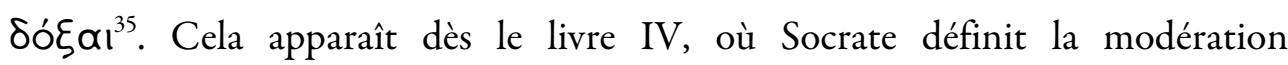

\footnotetext{
${ }^{35}$ Sur ce point, voir surtout J. MOLINE, art. cit., pp. 11 sq. La position de Moline est critiquée notamment par L. P. GERSON, op. cit., pp. 107-8, qui toutefois ne tient pas compte du texte du livre $\mathrm{X}$ analysé plus loin (Gerson cherche à contourner la difficulté p. 127, n. 46, d'une manière
}

PLATO, The electronic Journal of the International Plato Society, n 8, 2008. http://gramata.univ-paris1.fr/Plato

(c) All rights of reproduction of any form reserved. 


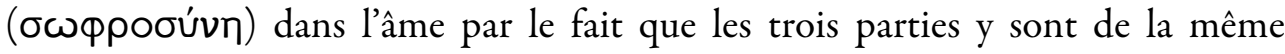


diriger (442 c9-d2) : pour pouvoir être de la même opinion, il faut d'abord que chacune soit capable d'avoir une opinion. D'ailleurs, le simple fait que cet accord doit résulter de la persuasion des parties inférieures par la partie supérieure suppose que les premières puissent avoir des $\delta$ ó $\alpha_{1}$ - correctes ou non. Mais le passage le plus significatif pour notre propos se trouve dans le livre X (602 c7-603 a8). Ce passage traite des illusions perceptives. La même grandeur, remarque Socrate, ne nous paraît pas égale selon qu'elle est vue de près ou de loin; et la situation est à peu près semblable avec les choses qui, selon qu'on les regarde dans l'eau ou hors de l'eau, nous paraissent courbées, droites, creuses ou bombées. Contre ce risque, nous disposons d'un secours précieux, à savoir le fait de mesurer,

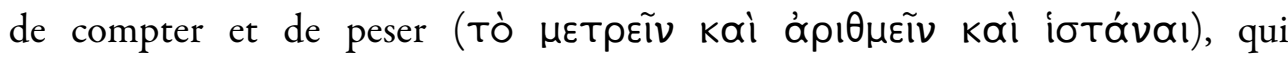

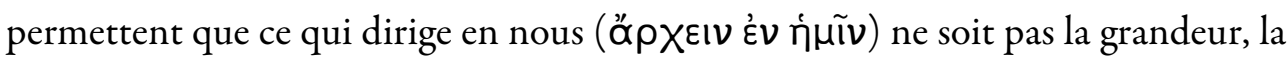

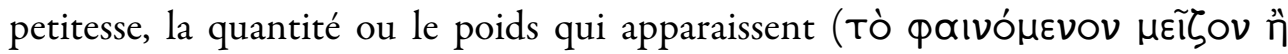

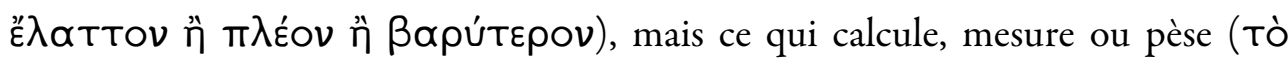

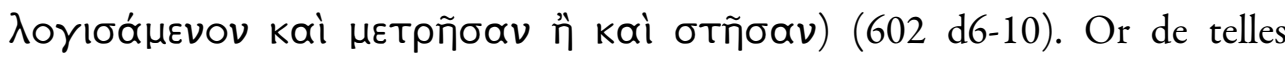

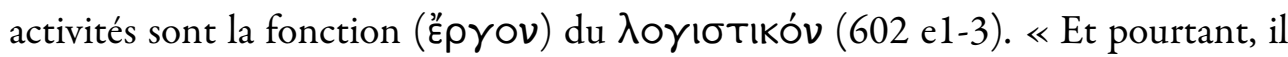
arrive bien souvent à celui qui a mesuré et signifié qu'une chose est plus grande ou plus petite qu'une autre, ou qu'elles sont égales, que le contraire lui apparaisse en même temps au sujet de ces mêmes choses »(602 e4-6). Or, rappelle Socrate, nous avons dit (dans le livre IV) qu'il est impossible à la même chose d'avoir en

qui me paraît entrer en contradiction tant avec le texte de ce passage qu'avec le principe de la

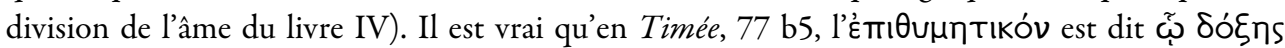

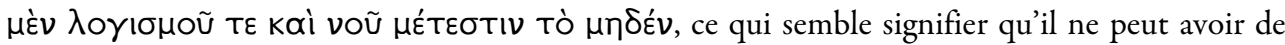

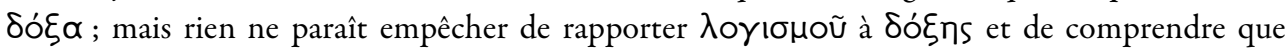

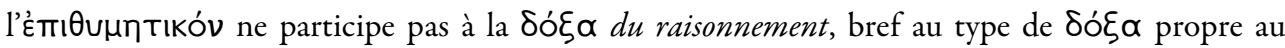


dialogue qui attribue quant à lui très clairement la $\delta$ óła à toutes les parties de l'âme, y compris l'Ėmı

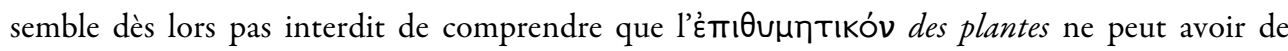
$\delta o ́ \xi \alpha$, sans que cela soit nécessairement le cas de celui des êtres humains.

PLATO, The electronic Journal of the International Plato Society, n 8, 2008.

http://gramata.univ-paris1.fr/Plato

(c) All rights of reproduction of any form reserved. 


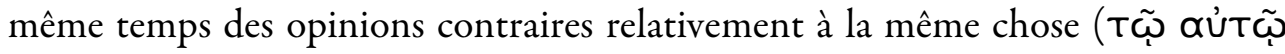

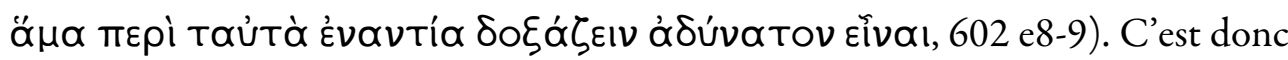
que la partie de l'âme qui opine contre la mesure n'est pas la même que celle qui

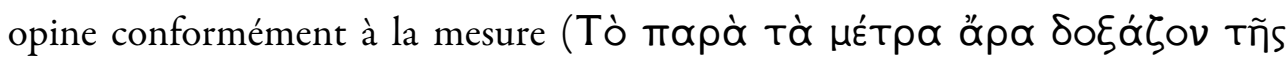

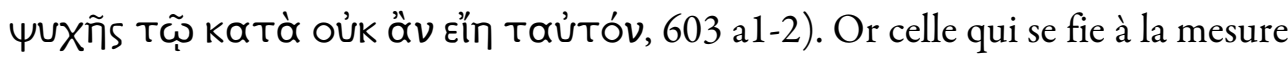

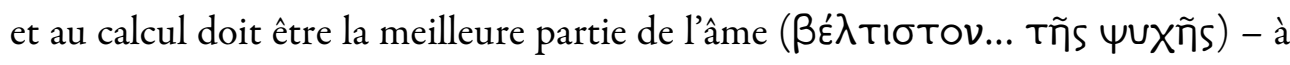

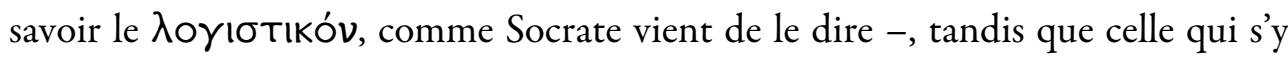
oppose doit appartenir à ce qui est de moins bonne qualité en nous ( $\tau \tilde{\omega} \nu$

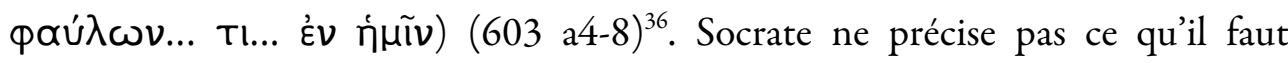
entendre par cette dernière expression, sans doute parce que tout ce passage a seulement pour but d'introduire une dernière critique de la poésie, en montrant que les illusions créées par celle-ci s'adressent aux deux parties inférieures de l'âme, suscitant non seulement des émotions telles que l'emportement et le chagrin, mais aussi le plaisir, et renforcent dès lors ces parties au détriment de la partie rationnelle (cf. 603 b7-607 b1). Cependant, dans le cas des illusions d'optique, le

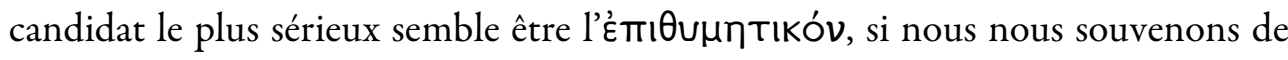
ce qui a été dit de son fonctionnement, à savoir qu'il est «aveugle » et

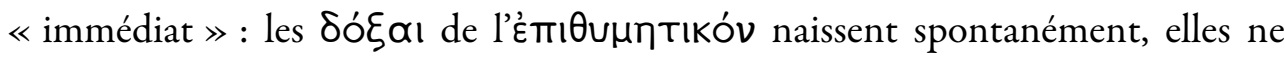
font que traduire le contenu de nos sensations immédiates. En revanche, les

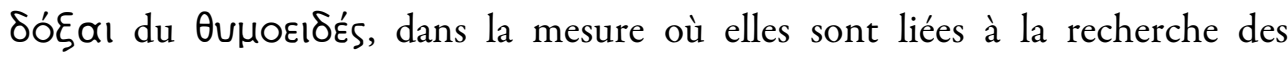
honneurs, sont avant tout des $\delta o ́ \xi \alpha ı$ collectives, héritées la plupart du temps de notre environnement social et culturel, qui nous enseigne ce qui est louable ou

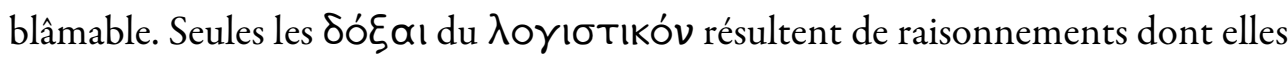

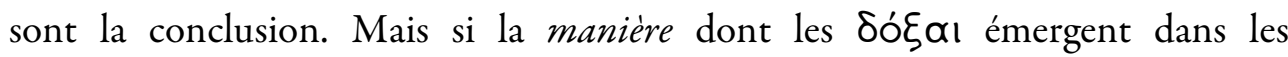

\footnotetext{
${ }^{36}$ Remarquons que ce passage permet de répondre à l'objection formulée par ARISTOTE dans le $D e$ Anima (III, 3, 428 b2-9) à l'encontre du lien établi par Platon entre $\phi \alpha v \tau \alpha \sigma i ́ \alpha$ et $\delta o ́ \xi \alpha$, à savoir que le soleil continue à nous apparaître avec une grandeur d'un pied même si nous savons par ailleurs qu'il est plus grand que la terre habitée. Pour Platon, cela n'implique pas que la

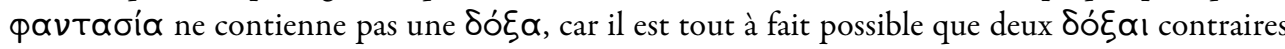
relatives au même objet cohabitent en nous; simplement, il faut les attribuer à deux parties de l'âme différentes.
}

PLATO, The electronic Journal of the International Plato Society, n 8, 2008. 
différentes parties de notre âme dépend du mode de fonctionnement de chacune de

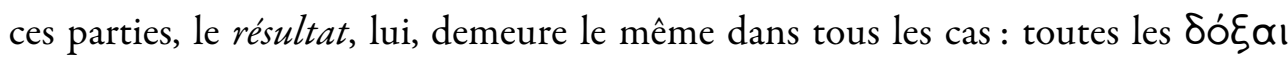
ont la même constitution, et celles qui résultent d'un raisonnement ne different pas par nature de celles qui naissent spontanément à la suite d'une sensation quelconque. Bien plus, toutes peuvent être soit vraies soit fausses, car tel est le lot

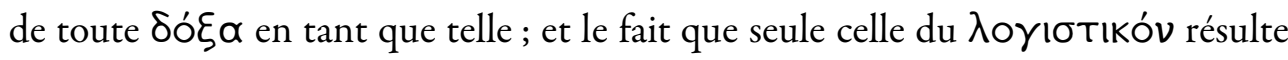
d'une recherche de la vérité ne garantit pas encore qu'elle l'a atteinte, tant que cette recherche ne s'est pas muée en science.

\section{c) La connaissance}

Aucune partie de l'âme n'est donc en propre l'«organe » de la $\delta o ́ \xi \alpha$, car celle-ci est compatible avec chacune des trois structures qui définissent ces parties.

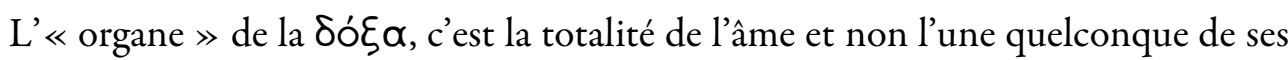
parties. La situation est toute différente dans le cas de la connaissance. En effet, il

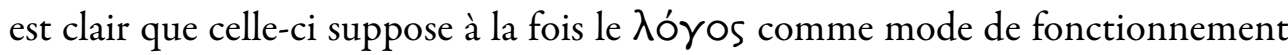
et la recherche de la vérité comme finalité. Elle ne peut donc être le fait que du $\lambda$ 入yıotıkóv. C'est d'ailleurs ce qui ressort du texte, qui désigne le sujet de la connaissance au moyen de différentes expressions - « la partie de l'âme à laquelle il appartient de s'attacher » à l'objet de connaissance, et qui en ce sens lui est

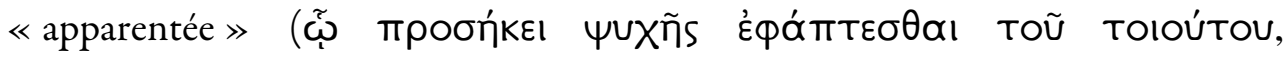

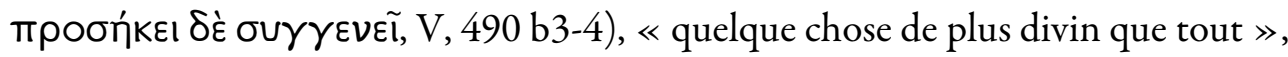

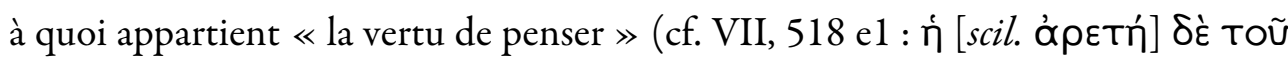

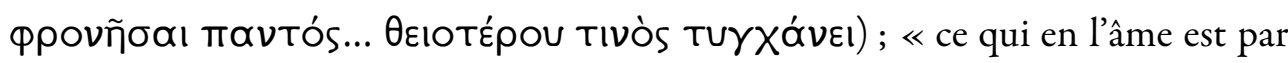

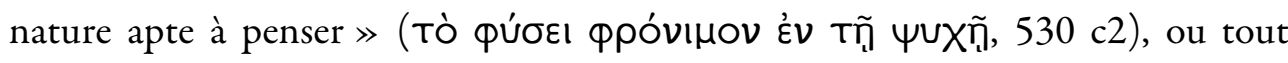

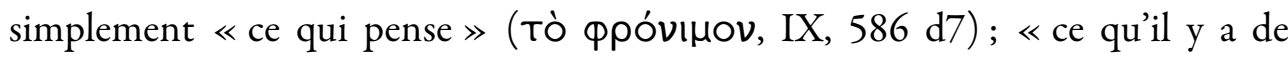

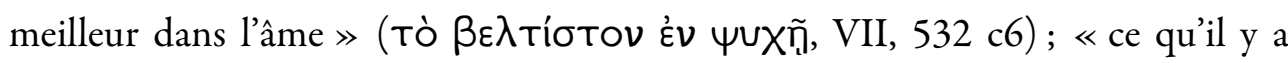

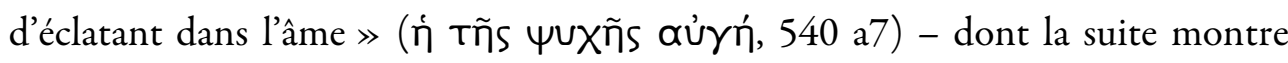

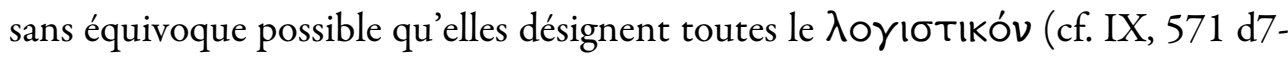

PLATO, The electronic Journal of the International Plato Society, n 8, 2008. http://gramata.univ-paris1.fr/Plato

(c) All rights of reproduction of any form reserved. 
572 e9, 589 d1, d7, e4, 590 c2, d1, d4, 591 a1, b4; X, 603 a4, 604 d4, 605 a10,

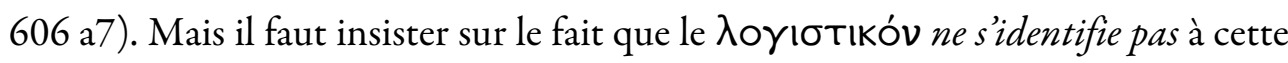
faculté qu'est la connaissance: il n'en est que l'organe (cf. Tò őpyavov फَ

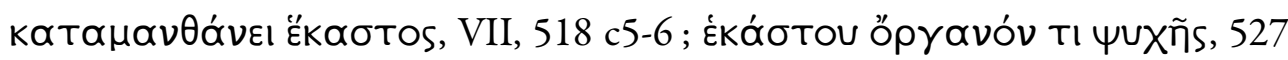
d8), correspondant à 《l'œil de l'âme » (Tò Tñs భUXñs ő $\mu \mu \alpha, 533$ d1-2). Or à la différence de l'œil du corps, qui a pour unique faculté la vue et qui, lorsqu'il se tourne vers ce qui est plongé dans l'obscurité, ne saisit tout simplement aucun objet, la partie rationnelle de l'âme a au moins deux facultés, puisque nous venons


pourquoi, lorsqu'elle se tourne non pas vers ces objets de connaissance que sont les Idées, mais vers le sensible, elle ne connaît pas, mais opine (cf. VI, 508 c4-d9).

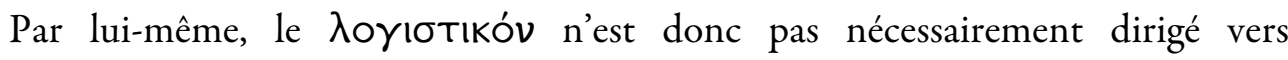
l'intelligible ${ }^{37}$, même s'il est vrai que, poussé jusqu'au bout de sa logique propre, son désir de vérité devrait naturellement le porter à désirer les seuls objets qu'il soit possible de penser avec une complète vérité, et dès lors à devenir philosophe plutôt que philodoxe (cf. V, 479 d10-480 a13). C'est le rôle de l'éducation

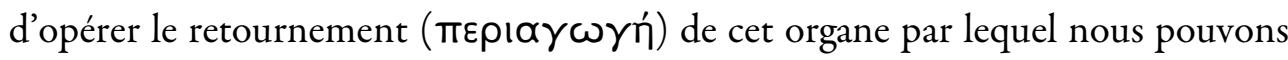
apprendre pour l'orienter vers ce qui est vraiment, retournement qui n'est possible que s'il est accompagné d'un retournement de l'âme tout entière, la débarrassant de ces « charges de plomb » que sont les plaisirs corporels en tout genre, qui la tirent constamment vers le bas (VII, 518 c4-519 b6).

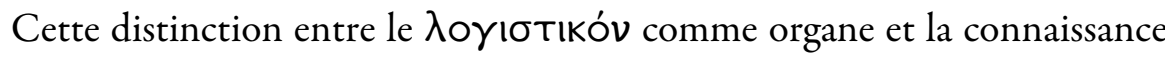
comme faculté de cet organe a une conséquence fondamentale, à savoir la

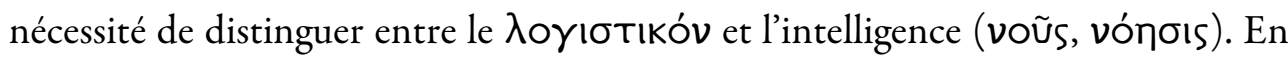

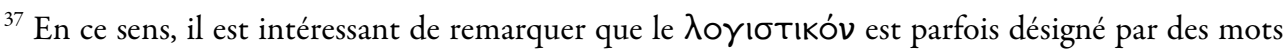

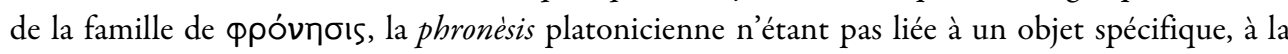
différence de l'intelligence, mais pouvant s'appliquer aussi bien au sensible qu'à l'intelligible, assurant ainsi la continuité entre ces deux plans, comme l'a montré M. DIXSAUT dans une conférence prononcée le 27 mai 2004 à l'Université de Louvain-la-Neuve et intitulée «Des multiples sens de la phronèsis dans les Dialogues de Platon ». Voir également du même auteur «De quoi les philosophes sont-ils amoureux ? Sur la phronèsis dans les Dialogues de Platon » [1997], repris dans Platon et la question de la pensée, Paris, 2000, pp. 93-119.
}

PLATO, The electronic Journal of the International Plato Society, n 8, 2008. http://gramata.univ-paris1.fr/Plato

(c) All rights of reproduction of any form reserved. 
effet, Socrate dit clairement que cette dernière n'apparaît que lorsque le $\lambda$ 入yı́tıkóv se dirige vers l'intelligible et donc connaît au sens propre du terme, tandis que lorsqu'il se contente d'émettre des opinions, elle demeure absente (cf. VI, 508 d3-9). Contrairement à une idée répandue, l'intelligence ne peut en

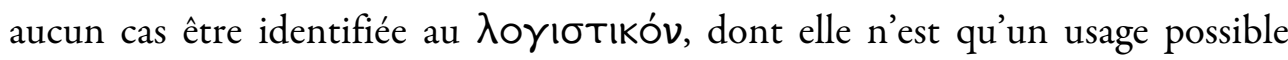
parmi d'autres. Cette conclusion, qui résulte de la République, vaut tout autant pour le Timée ${ }^{38}$, et elle est également compatible avec le Phèdre ${ }^{39}$. L'intelligence n'est pas une partie de l'âme, pas même une «partie » de cette partie qu'est le

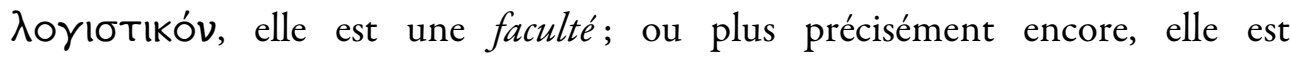
l'«actualisation »d'une faculté, à savoir la connaissance, car à la différence de cette dernière, qui est présente en nous de tout temps, l'intelligence est acquise,

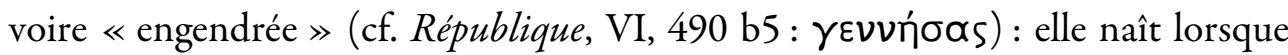

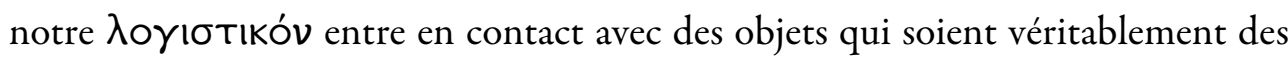
objets de connaissance, et qui dès lors la suscitent, puisqu'ils ne peuvent être saisis que par elle. Aussi étrange que cela puisse paraître, pour Platon, c'est la connaissance qui est la faculté dont l'intelligence est la mise en œuvre, et non

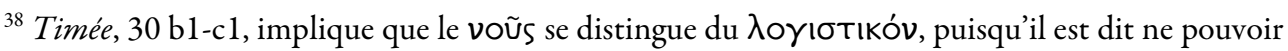

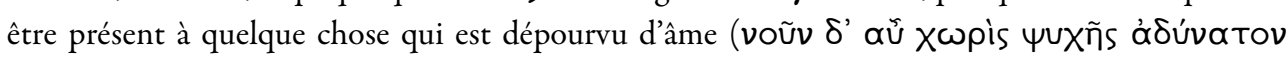

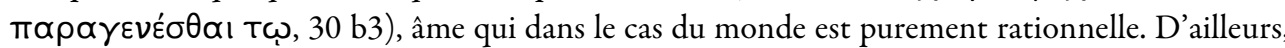

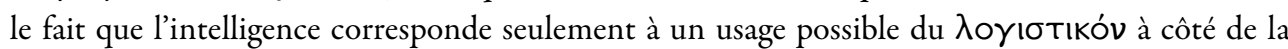
$\delta o ́ \xi \alpha$ est explicitement dit plus loin, lorsque intelligence et $\delta o ́ \xi \alpha$ sont représentées respectivement par les rotations en sens inverse des cercles du même et de l'autre (37 a2-c5). Ce

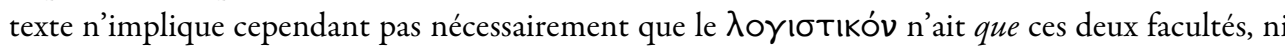

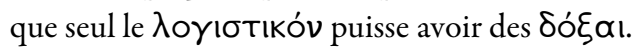

${ }^{39}$ Il pourrait toutefois sembler qu'en Phèdre, 247 c8, le terme voũs désigne le cocher, qui

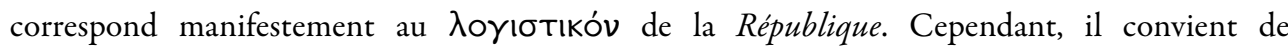

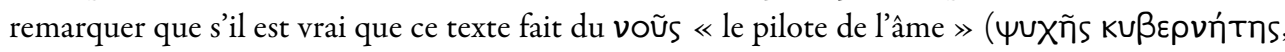
$247 \mathrm{c7}$ ), il ne le nomme pas « cocher » (ívíoxos); et tandis que le voũs est caractérisé par le fait de contempler l'intelligible, bref comme une faculté (ou plutôt comme son exercice), le cocher a également pour rôle de résister aux pulsions excessives de ses chevaux, ce qui correspond tout à fait à la description de la partie rationnelle de l'âme dans la République. Le problème de l'âme dont le destin est décrit dans le Phèdre est précisément que son cocher n'est pas assez fort pour se régler exclusivement sur ce «pilote» qu'est l'intelligence, ce qui cause la perte de ses ailes et sa « chute » dans le sensible.
}

PLATO, The electronic Journal of the International Plato Society, n 8, 2008.

http://gramata.univ-paris1.fr/Plato

(c) All rights of reproduction of any form reserved. 
l'inverse: l'intelligence, c'est la connaissance en tant qu'elle s'exerce effectivement. C'est d'ailleurs ce qui explique qu'au cours du livre VII, l'intelligence soit plus souvent désignée par le terme vónoıs que par celui de voũs, le suffixe -oıs servant généralement à indiquer une action ou un processus.

On voit ainsi qu'il n'existe aucune correspondance stricte entre parties de l'âme et facultés ${ }^{40}$ : non seulement une même faculté peut appartenir à différentes parties de l'âme, mais chaque partie peut avoir plusieurs facultés - ne fût-ce que la

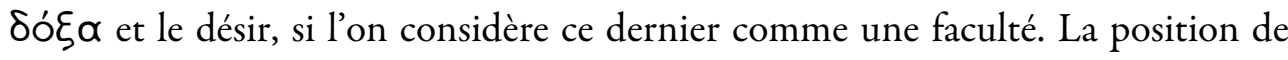
Platon se distingue dès lors tant de celle d'Aristote, qui semble ne pouvoir envisager la division de l'âme en parties que dans les termes d'une division en facultés $^{41}$, que de celle des stoïciens, qui assignent toutes les facultés à la partie

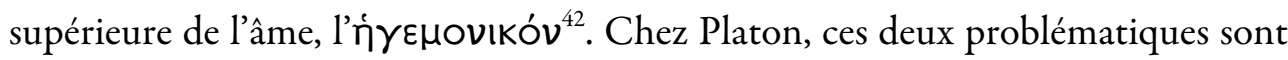
distinctes, ce qui explique que la question de la tripartition de l'âme n'intervienne pas dans l'étude des facultés que nous trouvons dans le Théétète et le Philébe. Même dans la République, où ces deux problématiques sont introduites pour la première fois, leur relation n'est pas étudiée systématiquement. Cela ne signifie pas pour autant qu'elles sont incompatibles; et comme souvent chez Platon, leur lieu de rencontre est la connaissance. En effet, cette faculté, du moins, n'appartient qu'à la partie rationnelle de l'âme. Dès lors, celui qui désire les objets de la connaissance, qui donc est philosophe au sens propre du terme, en mettant

${ }^{40}$ Contrairement à ce qu'écrit notamment A. GRAESER, op. cit., pp. 21-2, qui établit une

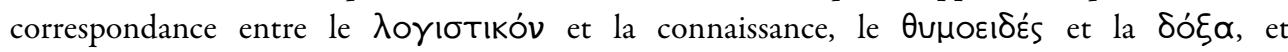

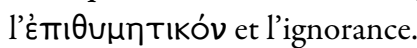

${ }^{41}$ Voir notamment De Anima, III, 10, 433 b1-4, où Aristote suppose cette identification entre

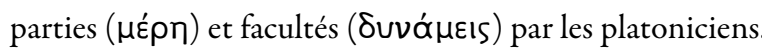

${ }^{42}$ Cf. J.-B. Gourinat, Les stö̈ciens et l'âme, Paris, 1996, p. 22. Une position comparable est attribuée à Platon, contre les textes me semble-t-il, par L. P. GERSON, qui écrit : «Selon mon

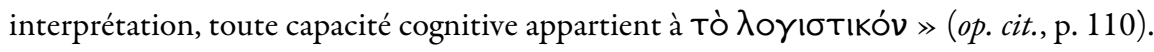

PLATO, The electronic Journal of the International Plato Society, n 8, 2008. http://gramata.univ-paris1.fr/Plato

(c) All rights of reproduction of any form reserved. 


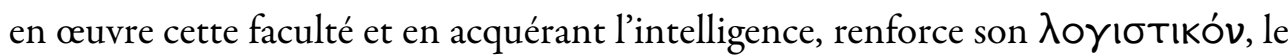
nourrit et établit sa suprématie sur les autres parties de son âme (cf. VIII, 550 b12, IX, 585 b6-c7). De cette manière, il réalise la justice en lui et établit un parfait accord entre les différentes parties de son âme. Cet accord interne est la vertu ('̊’ $\rho \tau$ Tn) de l'âme dans sa totalité, vertu qui lui permet d'accomplir au mieux sa

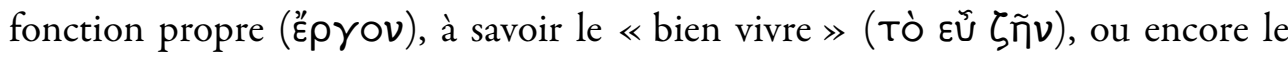
bonheur (cf. I, 352 d2-354 a9). Ainsi, dire que nous ne pouvons être heureux que

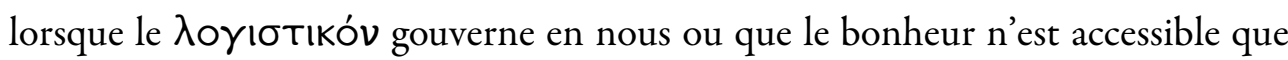
par la connaissance, c'est du pareil au même, puisque seule la connaissance peut

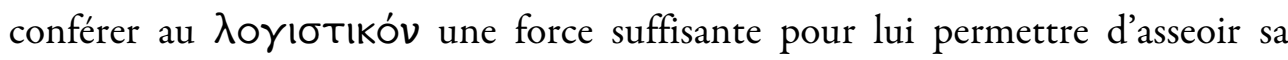
domination. C'est pourquoi au début du livre VI (484 a1-487 a8), Socrate peut montrer que le véritable philosophe tel qu'il a été défini à la fin du livre $\mathrm{V}$ dans le cadre de la distinction des facultés a nécessairement toutes les vertus qui résultent de la conception de la justice développée au livre IV dans le cadre de la tripartition de l'âme, établissant ainsi la jonction entre ces deux problématiques dont le simple besoin d'être conciliées manifeste le caractère a priori indépendant.

Sylvain DELCOMMINETTE

Université Libre de Bruxelles

PLATO, The electronic Journal of the International Plato Society, n 8, 2008. 